\title{
Prospects for precision measurement of diboson processes in the semileptonic decay channel in future LHC runs
}

\author{
Da Liu ${ }^{1}$ and Lian-Tao Wang ${ }^{2}$ \\ ${ }^{1}$ High Energy Physics Division, Argonne National Laboratory, Argonne, Illinois 60439, USA \\ ${ }^{2}$ Department of Physics, Enrico Fermi Institute, and Kavli Institute for Cosmological Physics, \\ University of Chicago, 5640 South Ellis Avenue, Chicago, Illinois 60637, USA
}

(Received 19 June 2018; published 5 March 2019)

\begin{abstract}
Precision measurements at the LHC are complementary to direct new physics searches. The high-energy distribution of diboson processes $[W W, W Z, V(W, Z) h]$ is a promising place, with the possibility of significant improvement in sensitivity as the data accumulates. We focus on the semileptonic final states and make projections of the reach for future runs of the LHC with integrated luminosities of $300 \mathrm{fb}^{-1}$ and $3 \mathrm{ab}^{-1}$. In the semileptonic channel of $W V$, we have employed a combination of kinematical distributions of both the $W$ and $Z$ and their decay products to select the longitudinally polarized $W$ and $Z$ to enhance the sensitivity. The measurement in this channel can surpass the sensitivity of the precision measurement at LEP, and they can be significantly more sensitive than the HL-LHC $h \rightarrow Z_{\gamma}$ measurements. Compared with the fully leptonic channel, the reach from the semileptonic channel can be better with effective suppression of the reducible background and systematic error. We also considered the reaches on the new physics mass scale in different new physics scenarios, including the strongly interacting light Higgs (SILH), the strongly coupled multipole interaction (Remedios), and the class of models with partially composite fermions.
\end{abstract}

DOI: 10.1103/PhysRevD.99.055001

\section{INTRODUCTION}

Precision measurement at the LHC will be one of its most important legacies. Electroweak symmetry breaking is one of the central questions of the Standard Model. Focusing on the electroweak sector of the Standard Model $(\mathrm{SM})$, precision measurements can provide valuable lessons which will help us address this question.

With the assumption that new physics particles would not be produced directly at the LHC, we parametrize their effect by a set of dimension-six effective field theory (EFT) operators [1-3]. In this paper, we focus on operators relevant to electroweak precision measurements. Such measurements on the $Z$-pole have been carried out at LEP [4], with typical precisions on the order of $10^{-3}$. Given that new physics effects are roughly of $\mathcal{O}\left(m_{Z}^{2} / \Lambda^{2}\right)$, this can be interpreted as constraining the scale of new physics to be higher than $\Lambda \sim 2 \mathrm{TeV}$. At the LHC, effects of new physics can potentially grow with energy. For example, if the leading effect is through interference between a dimension-6 operator and the SM, it could grow

Published by the American Physical Society under the terms of the Creative Commons Attribution 4.0 International license. Further distribution of this work must maintain attribution to the author(s) and the published article's title, journal citation, and DOI. Funded by SCOAP ${ }^{3}$. with energy as $\propto E^{2} / \Lambda^{2}$. In this case, since energies around $\mathrm{TeV}$ can be probed at the LHC, i.e., $E \sim 1 \mathrm{TeV}$, we only need $\sim 20 \%$ measurement of the cross section in this energy bin to achieve a reach on $\Lambda \sim 2 \mathrm{TeV}$ similar to that of LEP precision measurements. In order to fully take advantage of this effect, it is important to focus on final states whose amplitude not only grows like $E^{2}$, but also interferes with an approximately constant SM amplitude. In practice, this requires carefully designed cuts to select such final states. As we review in Sec. II, in the two-vector-boson channels ( $W W$ and $W Z$ ), an obvious channel would be the production of longitudinally polarized vector bosons. Polarization tagging would be crucial to separate it from channels with other polarizations. At the same time, such inference is guaranteed for the $V h$ channel.

The present bounds on these operators from LHC diboson processes have been studied in Refs. [5-14]. The prospects of probing these operators in the trilepton channel $W Z \rightarrow 3 \ell \nu$ and the dilepton channel $W W \rightarrow 2 \ell 2 \nu$ have been studied in Refs. [15,16], while the Higgsassociated production channels for the SM case have also been considered in Refs. $[17,18]$. In this paper, we focus our attention on the semileptonic channel of $W W, W Z$ production. In comparison with the pure leptonic channel, the semileptonic channel has a larger rate. At the same time, it presents new challenges. Not being able to clearly distinguish hadronically decaying $W$ and $Z$, we will have 
to consider them together. Unlike the $W Z$ channel, the $W W$ channel does not have the sharp "amplitude zero" feature in the central region. In this paper, we employ additional information from the distribution of the decay products of the vector boson to help tag its polarization. We have also included our analysis for the $V h$ channel, which is in broad agreement with the results in Ref. [15]. Based on this analysis, we make projections for the sensitivity to new physics.

The rest of this paper is organized as follows. In Sec. II, we describe the EFT framework of our analysis and offer general discussions of key aspects in the analysis of diboson channels. We present our analysis of the potential of the semileptonic channel, which is the main result of this paper, in Sec. III. In Sec. IV, we apply the result of our analysis to estimate reaches in the new physics scale in several more specific scenarios. Our conclusions are contained in Sec. V.

\section{THEORETICAL APPROACH}

After integrating out new physics, the SM Lagrangian is modified by the addition of higher-dimensional operators. We have

$$
\mathcal{L}=\mathcal{L}_{\mathrm{SM}}+\sum_{i} \frac{c_{i}}{\Lambda^{2}} \mathcal{O}_{i}+\cdots,
$$

where $\Lambda$ has no $\hbar$ dimension and it should be interpreted as a mass threshold. We have only included dimension-6 operators [1-3]. The operators most relevant for the diboson channel are

$$
\begin{aligned}
\mathcal{O}_{W} & =\frac{i g}{2}\left(H^{\dagger} \sigma^{a} \stackrel{\leftrightarrow}{D^{\mu}} H\right) D^{\nu} W_{\mu \nu}^{a} \quad \mathcal{O}_{B}=\frac{i g^{\prime}}{2}\left(H^{\dagger} \stackrel{\leftrightarrow}{D^{\mu}} H\right) \partial^{\nu} B_{\mu \nu}, \\
\mathcal{O}_{2 W} & =-\frac{1}{2} D^{\mu} W_{\mu \nu}^{a} D_{\rho} W^{a \rho \nu}, \quad \mathcal{O}_{2 B}=-\frac{1}{2} \partial^{\mu} B_{\mu \nu} \partial_{\rho} B^{\rho \nu}, \\
\mathcal{O}_{H W} & =i g\left(D^{\mu} H\right)^{\dagger} \sigma^{a}\left(D^{\nu} H\right) W_{\mu \nu}^{a}, \quad \mathcal{O}_{H B}=i g^{\prime}\left(D^{\mu} H\right)^{\dagger}\left(D^{\nu} H\right) B_{\mu \nu}, \\
\mathcal{O}_{3 W} & =\frac{1}{3 !} g \epsilon_{a b c} W_{\mu}^{a \nu} W_{\nu \rho}^{b} W^{c \rho \mu}, \quad \mathcal{O}_{T}=\frac{g^{2}}{2}\left(H^{\dagger} \stackrel{\leftrightarrow}{D}^{\mu} H\right)\left(H^{\dagger} \stackrel{\leftrightarrow}{D}^{\mu}\right) H, \\
\mathcal{O}_{R}^{u} & =i g^{\prime 2}\left(H^{\dagger} \stackrel{\leftrightarrow}{D^{\mu}} H\right) \bar{u}_{R} \gamma^{\mu} u_{R}, \quad \mathcal{O}_{R}^{d}=i g^{2}\left(H^{\dagger} \stackrel{\leftrightarrow}{D^{\mu}} H\right) \bar{d}_{R} \gamma^{\mu} d_{R}, \\
\mathcal{O}_{L}^{q} & =i g^{2}\left(H^{\dagger} \stackrel{\leftrightarrow}{D^{\mu}} H\right) \bar{q}_{L} \gamma^{\mu} q_{L}, \quad \mathcal{O}_{L}^{(3) q}=i g^{2}\left(H^{\dagger} \sigma^{a} \stackrel{\leftrightarrow}{D^{\mu}} H\right) \bar{q}_{L} \sigma^{a} \gamma^{\mu} q_{L},
\end{aligned}
$$

where $H^{\dagger} \stackrel{\leftrightarrow}{D^{\mu}} H \equiv H^{\dagger} D_{\mu} H-\left(D_{\mu} H\right)^{\dagger} H$. From this list, we will not further consider $T$-parameter operator $\mathcal{O}_{T}$. It has been well constrained by LEP experiment, and it is unlikely that LHC measurement can reach a comparable level. We have also not included the operator $\mathcal{O}_{H}=\frac{1}{2 f^{2}}\left(\partial|H|^{2}\right)^{2}$ in the list. It modifies the Higgs gauge boson coupling. Current results of Higgs coupling measurement have already constrained $f \gtrsim 800 \mathrm{GeV}[19,20]$, and the precision can reach $f \gtrsim 1200 \mathrm{GeV}$ with the HL-LHC [21-23]. It will lead to strong $W_{L} W_{L}(h h)$ scattering, as dictated by the Goldstone equivalent theorem [24,25]. However, the effect is more prominent at higher energies $\sim(f / v)^{2} \times \mathrm{TeV}$. The sensitivities of LHC to $\mathcal{O}_{H}$ in the diboson channels are weak, reaching $f \gtrsim 350 \mathrm{GeV}$ at the HL-LHC in the double Higgs final states [26] and $f \gtrsim 550 \mathrm{GeV}$ at the HL-LHC in the same sign dilepton channel of $W^{ \pm} W^{ \pm}$[27]. It cannot compete with the Higgs coupling measurement.

The contributions of these operators to scattering amplitudes depend on the final states. We will consider the so-called diboson processes $q \bar{q} \rightarrow V_{1} V_{2}$ and $q \bar{q} \rightarrow V h$, where $V=W^{ \pm}, Z$. With our normalization, the largest $\mathrm{SM}$ amplitude is a constant. ${ }^{1}$ For dimension- 6 operators,

\footnotetext{
${ }^{1}$ There are also t-channel poles in the forward region; here for simplicity we are focusing on the central region.
}

their contributions to the amplitudes can grow at most as $E^{2}$. Hence, we should look for a channel with interference between the SM and the new physics amplitude growing as $E^{2}$, or at least growing with energy. In order to have the energy growing behavior, it is not enough to just have the contribution of dimension- 6 operators to the amplitude to grow with energy. It is crucial to have the corresponding SM amplitude not decreasing at least as fast with energy. This condition can in principle be relaxed if the SM background interfering with the signal is the only SM background. In this case, we can have good sensitivity as long as $S / \sqrt{B}$ grows with energy. A SM background decrease with energy can in principle satisfy this condition, even if the interference piece does not grow. However, in practice, such cases are difficult to find. There are almost always (ir)reducible SM backgrounds which do not decrease with energy. Hence, the channels which have the interference piece growing with energy remain our best hope. ${ }^{2}$

Perhaps the most straightforward cases to consider are the $W h$ and $Z h$ channels. In this case, the new physics

\footnotetext{
${ }^{2}$ In this paper, we will simply extract the expected sensitivity on the new physics effects by combining the different energy bins; see Ref. [28] for a more dedicated study on obtaining the optimal estimations of the dimension-six deformations on the differential distributions of the SM observables.
} 
TABLE I. High-energy behavior for the helicity amplitudes $q \bar{q} \rightarrow W^{+} W^{-}$, where we omit the gauge couplings $g^{2}, g^{\prime 2}$ in front of the amplitudes [32]. $\mathcal{O}_{2 W, 2 B}$ has similar behavior as $\mathcal{O}_{W, B}$. $E$ can be thought as half of the partonic center-of-mass energy (i.e., the energy of a single $W$ boson). For the zeros in the table, the corresponding amplitudes are zero in the zero mass limit. For the $W Z$ final state, the only nonzero amplitudes are those from the left-handed quarks. In addition, only the $\mathcal{O}_{W, H W}$ operators have energy growing behavior in the purely longitudinal helicity state.

\begin{tabular}{lcccccc}
\hline \hline$\left(h_{W^{+}}, h_{W^{-}}\right)$ & $\mathrm{SM}$ & $\mathcal{O}_{W}$ & $\mathcal{O}_{H W}$ & $\mathcal{O}_{B}$ & $\mathcal{O}_{H B}$ & $\mathcal{O}_{3 W}$ \\
\hline$( \pm, \mp)$ & 1 & 0 & 0 & 0 & 0 & 0 \\
$(0,0)$ & 1 & $\frac{E^{2}}{\Lambda^{2}}$ & $\frac{E^{2}}{\Lambda^{2}}$ & $\frac{E^{2}}{\Lambda^{2}}$ & $\frac{E^{2}}{\Lambda^{2}}$ & 0 \\
$(0, \pm),( \pm, 0)$ & $\frac{m_{W}}{E}$ & $\frac{E m_{W}}{\Lambda^{2}}$ & $\frac{E m_{W}}{\Lambda^{2}}$ & $\frac{E m_{W}}{\Lambda^{2}}$ & $\frac{E m_{W}}{\Lambda^{2}}$ & $\frac{E m_{W}}{\Lambda^{2}}$ \\
$( \pm, \pm)$ & $\frac{m_{W}^{2}}{E^{2}}$ & $\frac{m_{W}^{2}}{\Lambda^{2}}$ & $\frac{m_{W}^{2}}{\Lambda^{2}}$ & $\frac{m_{W}^{2}}{\Lambda^{2}}$ & 0 & $\frac{E^{2}}{\Lambda^{2}}$ \\
& & $q_{R} \bar{q}_{L} \rightarrow W^{+} W^{-}$ & & \\
$( \pm, \mp)$ & 0 & 0 & 0 & 0 & 0 & 0 \\
$(0,0)$ & 1 & $\frac{m_{W}^{2}}{\Lambda^{2}}$ & $\frac{m_{W}^{2}}{\Lambda^{2}}$ & $\frac{E^{2}}{\Lambda^{2}}$ & $\frac{E^{2}}{\Lambda^{2}}$ & 0 \\
$(0, \pm),( \pm, 0)$ & $\frac{m_{W}}{E}$ & $\frac{m_{W}^{2} m_{Z}^{2}}{\Lambda^{2} E^{2}}$ & $\frac{E m_{W}}{\Lambda^{2}}$ & $\frac{E m_{W}}{\Lambda^{2}}$ & $\frac{E m_{W}}{\Lambda^{2}}$ & $\frac{m_{W}^{2} m_{Z}^{2}}{\Lambda^{2} 2^{2}}$ \\
$( \pm, \pm)$ & $\frac{m_{W}^{2}}{E^{2}}$ & $\frac{m_{W}^{2}}{\Lambda^{2}}$ & $\frac{m_{W}^{2}}{\Lambda^{2}}$ & $\frac{m_{W}^{2}}{\Lambda^{2}}$ & 0 & $\frac{m_{W}^{2}}{\Lambda^{2}}$ \\
\hline \hline
\end{tabular}

amplitude interferes with the full Standard Model amplitude. The only challenge would be to identify the final states amid the reducible SM backgrounds. This has been demonstrated to be feasible [29]. In particular, boost techniques play an important role in separating signal from reducible background [30,31]. At the same time, the boosted regime is also precisely the place for enhancing the new physics effect. Further studies of this channel have been presented recently $[17,18]$.

The channels with two vector gauge bosons are more complicated. In Table I, we have shown the high-energy behavior for the helicity amplitudes of the process $q \bar{q} \rightarrow$ $W^{+} W^{-}$in the SM case and also the contribution from dimension-six operators. We have omitted the gauge coupling squares $g^{2}, g^{\prime 2}$ in front of the amplitudes [32]. From Table I (see also [33]), we conclude that the most promising channels are those with longitudinally polarized vector bosons, as the interference piece grows with energy as $\propto E^{2}$. Hence, we expect that isolating events with longitudinal polarized vector bosons will be particularly important. There can be two strategies in achieving this goal. One is to take advantage of the fact that final states with different polarizations have different kinematical distribution [32]. A particularly useful example is the so-called "amplitude zero" in the transversely polarized $W Z$ final states [34,35]. In this case, using kinematical cuts which select the central region enhances the longitudinally polarized component. This approach has been used in Ref. [15].

The second strategy is directly tagging the polarization of a gauge boson from the angular distribution of its decay products. Such a polarization tagging can be challenging. The basic difference would be in the angular distribution of
TABLE II. Observables for probing the higher-dimensional operators. $c_{f}$ denotes the Wilson coefficients of the fermionic operators in Eq. (2). For reference, we have also included contributions from potential dimension-8 operators with Wilson coefficients denoted by $c_{T X}$. See Appendix C of Ref. [37] for the definition of the dimension-8 operators.

\begin{tabular}{lc}
\hline \hline Observable & $\delta O / O_{\mathrm{SM}}$ \\
\hline$W_{L}^{+} W_{L}^{-}$ & {$\left[\left(c_{W}+c_{H W}-c_{2 W}\right) T_{f}^{3}+\left(c_{B}+c_{H B}-c_{2 B}\right) Y_{f} t_{w}^{2}\right] \frac{E^{2}}{\Lambda^{2}}, c_{f} \frac{E^{2}}{\Lambda^{2}}$} \\
$W_{T}^{+} W_{T}^{-}$ & $c_{3 W} \frac{m_{W}^{2}}{\Lambda^{2}}+c_{3 W}^{2} \frac{E^{4}}{\Lambda^{4}}, c_{T W W} \frac{E^{4}}{\Lambda^{4}}$ \\
$W_{L}^{ \pm} Z_{L}$ & $\left(c_{W}+c_{H W}-c_{2 W}+4 c_{L}^{(3) q}\right) \frac{E^{2}}{\Lambda^{2}}$ \\
$W_{T}^{ \pm} Z_{T}(\gamma)$ & $c_{3 W} \frac{m_{W}^{2}}{\Lambda^{2}}+c_{3 W}^{2} \frac{E^{4}}{\Lambda^{4}}, c_{T W B} \frac{E^{4}}{\Lambda^{4}}$ \\
$W_{L}^{ \pm} h$ & $\left(c_{W}+c_{H W}-c_{2 W}+4 c_{L}^{(3) q}\right) \frac{E^{2}}{\Lambda^{2}}$ \\
$Z h$ & $\left(c_{W}+c_{H W}-c_{2 W}\right) T_{f}^{3}-\left(c_{B}+c_{H B}-c_{2 B}\right) Y_{f} t_{W}^{2} \frac{E}{2}_{\frac{L^{2}}{2}}, c_{f} \frac{E^{2}}{\Lambda^{2}}$ \\
$Z_{T} Z_{T}$ & $\left(c_{T W W}+t_{w}^{4} c_{T B B}-2 T_{f}^{3} t_{w}^{2} c_{T W B}\right) \frac{E^{4}}{\Lambda^{4}}$ \\
$\gamma \gamma$ & $\left(c_{T W W}+c_{T B B}+2 T_{f}^{3} c_{T W B}\right) \frac{E^{4}}{\Lambda^{4}}$ \\
$\hat{S}$ & $\left(c_{W}+c_{B}\right) \frac{m_{W}^{2}}{\Lambda^{2}}$ \\
$h \rightarrow Z \gamma$ & $\left(c_{H W}-c_{H B}\right) \frac{(4 \pi v)^{2}}{\Lambda^{2}}$ \\
$h \rightarrow W^{+} W^{-}$ & $\left(c_{W}+c_{H W}\right) \frac{m_{W}^{2}}{\Lambda^{2}}$ \\
\hline \hline
\end{tabular}

the decay product in the rest frame of the gauge boson. Even with perfect reconstruction and identification, one would not expect the difference between different polarizations to be much more than order 1 . In practice, one strategy would be to reconstruct the rest frame of the gauge boson, and use the angular distribution of the decay product [36]. The systematic error in the reconstruction needs to be taken into account. Another strategy would be to use the kinematical feature of the decay product in the lab frame. This has the advantage of skipping the step of reconstructing the rest frame of the gauge boson. However, some of the information of the angular distribution will be washed out.

A list of diboson channels and other observables, and the contributions from new physics operators, are presented in Table II. For reference, we have also included the contribution of dimension-8 operators, where we refer to Appendix $\mathrm{C}$ of Ref. [37] for the definitions. $\delta O / O_{\mathrm{SM}}$ denotes the ratios between the contribution to the cross sections or decay widths from higher-dimension operators and the SM prediction. We see that each of the observables receives contributions from multiple operators. More specifically, the contributions to diboson production in the highenergy limit depend on the following combinations [15]:

$$
\begin{aligned}
& c_{q_{L}}^{(3)}=c_{W}+c_{H W}-c_{2 W}+4 c_{L}^{(3) q}, \\
& c_{u_{L}}^{(1)}=c_{B}+c_{H B}-c_{2 B}+4 c_{L}^{q}, \\
& c_{d_{L}}^{(1)}=c_{B}+c_{H B}-c_{2 B}-4 c_{L}^{q}, \\
& c_{u_{R}}^{(1)}=c_{B}+c_{H B}-c_{2 B}+3 c_{u_{R}}, \\
& c_{d_{R}}^{(1)}=c_{B}+c_{H B}-c_{2 B}-6 c_{d_{R}} .
\end{aligned}
$$


This result can be understood easily by using the following operator relations (together with additional equations of motion) to rewrite operators $\mathcal{O}_{H W, H B}, \mathcal{O}_{W, B}, \mathcal{O}_{2 W, 2 B}$ in terms of the operators with more fields, such as $\mathcal{O}_{W W, W B, B B}, \mathcal{O}_{L, R}^{f}, \mathcal{O}_{4 f}, \mathcal{O}_{y_{f}}$, and so on $[2,3]$ :

$$
\begin{aligned}
\mathcal{O}_{B} & =\mathcal{O}_{H B}+\mathcal{O}_{B B}+\frac{1}{4} \mathcal{O}_{W B}, \quad \mathcal{O}_{W B}=g g^{\prime}\left(H^{\dagger} \sigma^{a} H\right) W_{\mu \nu}^{a} B^{\mu \nu}, \quad \mathcal{O}_{B B}=g^{\prime 2} H^{\dagger} H B_{\mu \nu} B^{\mu \nu} \\
\mathcal{O}_{W} & =\mathcal{O}_{H W}+\frac{1}{4} \mathcal{O}_{W W}+\frac{1}{4} \mathcal{O}_{W B}, \quad \mathcal{O}_{W W}=g^{2} H^{\dagger} H W_{\mu \nu}^{a} W^{a \mu \nu} \\
\mathcal{O}_{4 f} & =\bar{f} \gamma^{\mu} f \bar{f} \gamma_{\mu} f, \quad \mathcal{O}_{y_{f}}=y_{f} H^{\dagger} H \bar{f}_{L} H f_{R} .
\end{aligned}
$$

The resulting set of operators is called the Warsaw basis [1]. For example, from the first relation on the first line of Eq. (4), the operators $\mathcal{O}_{B}$ and $\mathcal{O}_{H B}$ contribute in the same way to longitudinal diboson final states, since $\mathcal{O}_{W B}$ and $\mathcal{O}_{B B}$ only contribute to the production of the transverse diboson final states. Similarly, the operator $\mathcal{O}_{B}$ can be related to the $\mathcal{O}_{L, R}^{f}$ operators by equations of motion of the hypercharge gauge field.

It is impossible to distinguish separate contributions from operators within each combination from diboson measurement. Besides diboson production, one of the most important observables is the oblique $S$-parameter [38], which has been well constrained by LEP precision electroweak measurement [4]. It depends on a different combination of the operators $\mathcal{O}_{W}+\mathcal{O}_{B}$. Therefore, it is complementary to the diboson measurement at the LHC. At the same time, we do not expect large cancellation among operators short of large fine-tuning or special symmetry. In this case, we can view the LEP measurement of the $S$-parameter as setting a generic limit on the size of $\mathcal{O}_{W}$ and $\mathcal{O}_{B}$ and use that as a target for the LHC experiments. A similar argument also applies to the measurement of Higgs rare decay $h \rightarrow Z \gamma$ at the HL-LHC, which will be sensitive to the operator combination $\mathcal{O}_{H W}-\mathcal{O}_{H B}$. For this measurement at the HL-LHC, we will use the projections made in Ref. [23].

So far, our discussion is at the level of the parton level cross section. The observable cross section is obtained after convolution with parton distribution functions. Taking this into account, the signal cross section scales with energy as

$$
\sigma_{\text {sig }} \propto \mathcal{M}_{\mathrm{SM}}\left(\frac{E}{\Lambda}\right)^{d-4}\left(\frac{1}{E}\right)^{n_{L}+2}
$$

where $d$ is the dimension of the EFT operator responsible for the signal, and $\mathcal{M}_{\mathrm{SM}}$ is the SM amplitude with which the new physics amplitude interferes. $n_{L}$ parametrizes the dependence of parton luminosity on the parton center-ofmass energy. Parton luminosity is a sharp falling function of $E$. Typically, $n_{L}$ is a large power, around 4-6. If the search channel is statistics dominated, we have

$$
\frac{S}{\sqrt{B}} \propto\left(\frac{E}{\Lambda}\right)^{d-4}\left(\frac{1}{E}\right)^{n_{L} / 2+1} \times \sqrt{\mathcal{L}},
$$

where $\mathcal{L}$ is the integrated luminosity. To obtain this qualitatively scaling behavior, we have made the crude approximation that $\sigma_{\mathrm{bkg}} \sim\left|\mathcal{M}_{\mathrm{SM}}\right|^{2}$. This means the sensitivity of different energy bins depends on the dimension of the EFT operator to be probed. For example, for $d=6$ operators in Eq. (2), lower energy bins have higher sensitivity. On the other hand, for probing $d=8$ EFT operators, we expect that higher energy bins yield better sensitivity. However, the assumption of statistics domination is certainly not realistic. Systematic error is very important, particularly for precision measurements. Lower energy bins, typically with a smaller $S / B$, will be more affected (and sometimes dominated) by systematics. Therefore, in reality, the most sensitive energy bin is typically determined by a trade-off between systematics and statistics.

\section{PHENOMENOLOGY OF DIBOSON PROCESSES IN THE SEMILEPTONIC CHANNEL}

We will focus on the following semileptonically decaying channels at the LHC:

$$
\begin{aligned}
p p & \rightarrow W V \rightarrow \ell \nu q \bar{q}, \quad p p \rightarrow W h \rightarrow \ell \nu b \bar{b}, \\
p p & \rightarrow Z h \rightarrow \ell^{+} \ell^{-} b \bar{b}, \quad p p \rightarrow Z h \rightarrow \nu \bar{\nu} \bar{b},
\end{aligned}
$$

where $\ell=e, \mu$ and $V=W, Z$.

For the Monte Carlo simulation, we first implement the dimension-six operators in Eq. (2) in a UFO model by using FeynRules [39]. We then use MadGraph5 [40] to simulate the signal and background events at leading order (LO). The cross sections of the processes considered in this paper are also calculated using MadGraph5 at the LO. For the studies in this paper, we have used NNPDF 2.3LO1 [41] as the parton distribution functions.

\section{A. $W V$ processes}

We start from the semileptonic final states from the $W V$ processes. The longitudinal modes of $W V$ tend to be produced more centrally than the transverse ones. Two possible kinematical variables which can capture this 

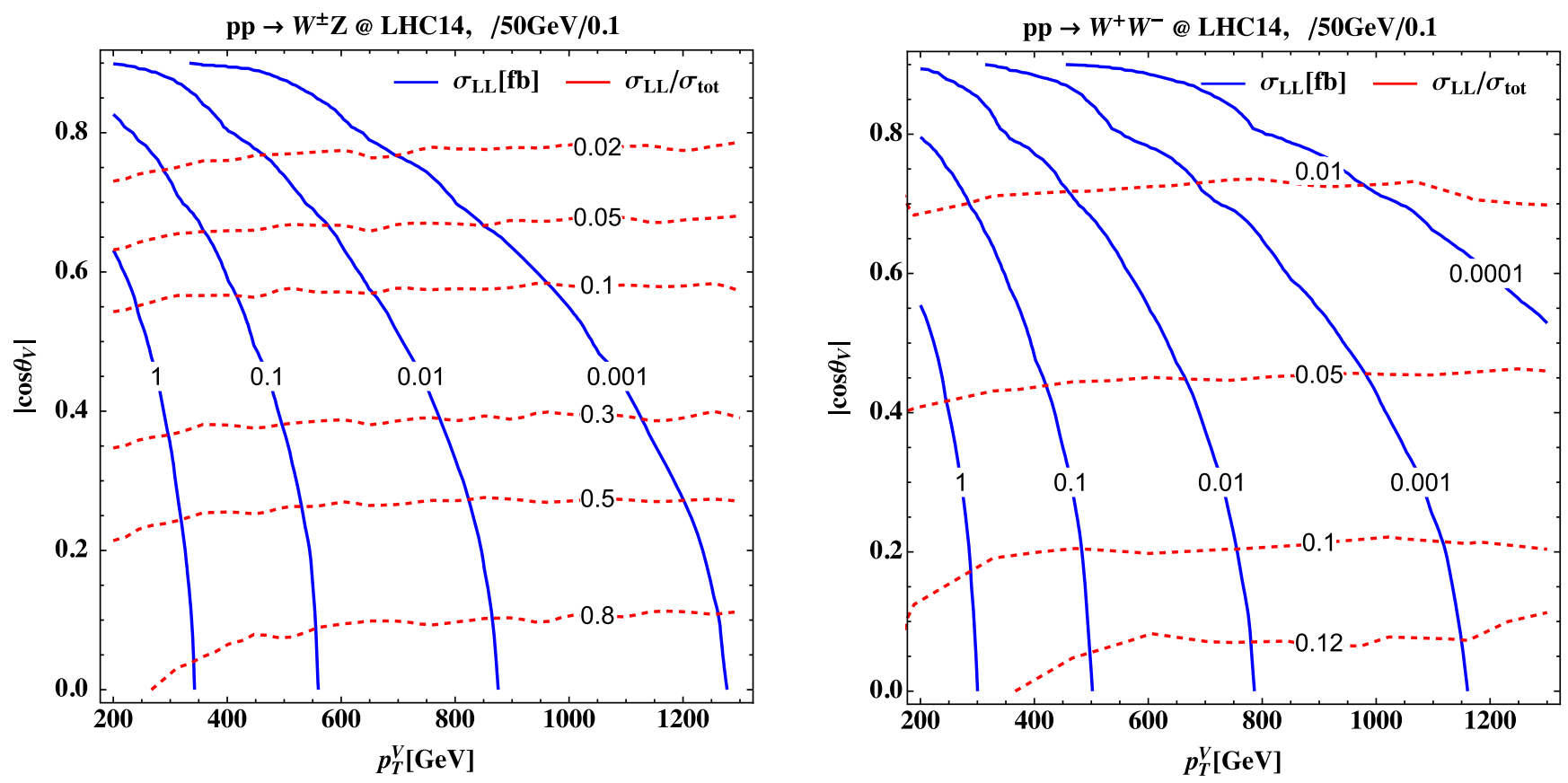

FIG. 1. Contours of production cross section of longitudinally polarized vector bosons $\sigma_{L L}$ and its ratio to the total cross section, $\sigma_{L L} / \sigma_{\text {tot }}$, in the $\left|\cos \theta_{V}\right|-p_{T}^{V}$ plane. $\theta_{V}$ is the scattering angle in the parton center-of-mass frame. The left (right) panel is for $W Z$ ( $W W$ ) production. We require $\left|\eta_{V}\right|<2.5$.

feature are the transverse momentum, $p_{T}^{V}$, and the scattering angle in the parton center-of-mass frame, $\theta_{V}$, of the vector bosons. In Fig. 1, we plot the contours of the production cross section of longitudinally polarized vector bosons $\sigma_{L L}$, and its ratio to the total cross section, $\sigma_{L L} / \sigma_{\text {tot }}$, in the $\left|\cos \theta_{V}\right|-p_{T}^{V}$ plane. We see that the $W_{L} Z_{L}$ can be dominant in the central region, while $W_{L} W_{L}$ is at most $10 \%$ of the total rate. This is due to the presence (absence) of the so-called "amplitude zero" in $W Z(W W)$ channels [34]. The behavior of the contours can be understood qualitatively. In the high-energy regime, we can approximately neglect effects of the gauge boson masses $m_{W, Z}$. The differential production cross section for vector bosons with helicity $h_{V 1}$ and $h_{V 2}$ from initial parton $i$ and $j$ is

$\frac{d^{2} \sigma^{h_{V 1} h_{V 2}}}{d p_{T}^{V} d \cos \theta_{V}}=\frac{1}{128 \pi N_{c}} \sum_{i j} \frac{\beta}{E^{2}} \frac{d L_{i j}}{d E} \frac{d E}{d p_{T}^{V}} \times\left|\mathcal{M}_{i j}^{h_{V 1}} h_{V 2}\left(\theta_{V}, E\right)\right|^{2}$

where $E$ is the energy for the single vector boson in the partonic center-of-mass frame. We have

$$
p_{T}^{V}=p \sin \theta_{V}, \quad \beta=\frac{p}{E}, \quad \frac{d E}{d p_{T}^{V}}=\frac{p_{T}^{V}}{E \sin ^{2} \theta_{V}}=\frac{\beta}{\sin \theta_{V}},
$$

where $p=|\vec{p}|$ denotes the magnitude of the three-momentum of the gauge boson in the partonic center-of-mass frame. For simplicity, we define the helicity states in the partonic center-of-mass frame. $\frac{d L_{i j}}{d E}$ is the parton luminosity defined as

$$
\frac{d L_{i j}}{d E}=\frac{8 E}{S} \int_{s / S}^{1} \frac{d x}{x} f_{i}(x, \mu) f_{j}(s / S x, \mu), \quad s=4 E^{2},
$$

where $s$ denotes the square of the partonic center-of-mass energy and $S$ means the proton-proton center-of-mass energy squared. First, we consider the $W W$ production. To get a qualitative understanding, we can ignore the contribution from hypercharge gauge coupling since it is small in comparison with the $S U(2)_{L}$ contribution. In the high-energy limit, we have

$$
\begin{aligned}
\left|\mathcal{M}_{W W}^{T T}\right|^{2} & =\frac{g^{4}}{32}\left(\frac{s^{2}}{t^{2}}+\frac{s^{2}}{u^{2}}\right) \sin ^{2} \theta_{V}\left(1+\cos ^{2} \theta_{V}\right), \\
\left|\mathcal{M}_{W W}^{L L}\right|^{2} & =\frac{g^{4}}{32} \sin ^{2} \theta_{V}
\end{aligned}
$$

where the amplitudes are summed over initial states $u \bar{u}$ and $\bar{u} u$. These are even functions of $\cos \theta_{V}$. Including the contribution from $d \bar{d}+\bar{d} d$ does not change the form of the squared amplitudes. Thus, the parton luminosity can be factored out, and the ratio $d^{2} \sigma_{W W}^{L L} / d^{2} \sigma_{W W}^{T T}$ only depends on the ratio of the squared amplitudes

$$
\frac{d^{2} \sigma_{W W}^{L L}}{d^{2} \sigma_{W W}^{T T}} \sim \frac{1}{8} \frac{\left(1-\cos ^{2} \theta_{V}\right)^{2}}{\left(1+\cos ^{2} \theta_{V}\right)^{2}} .
$$



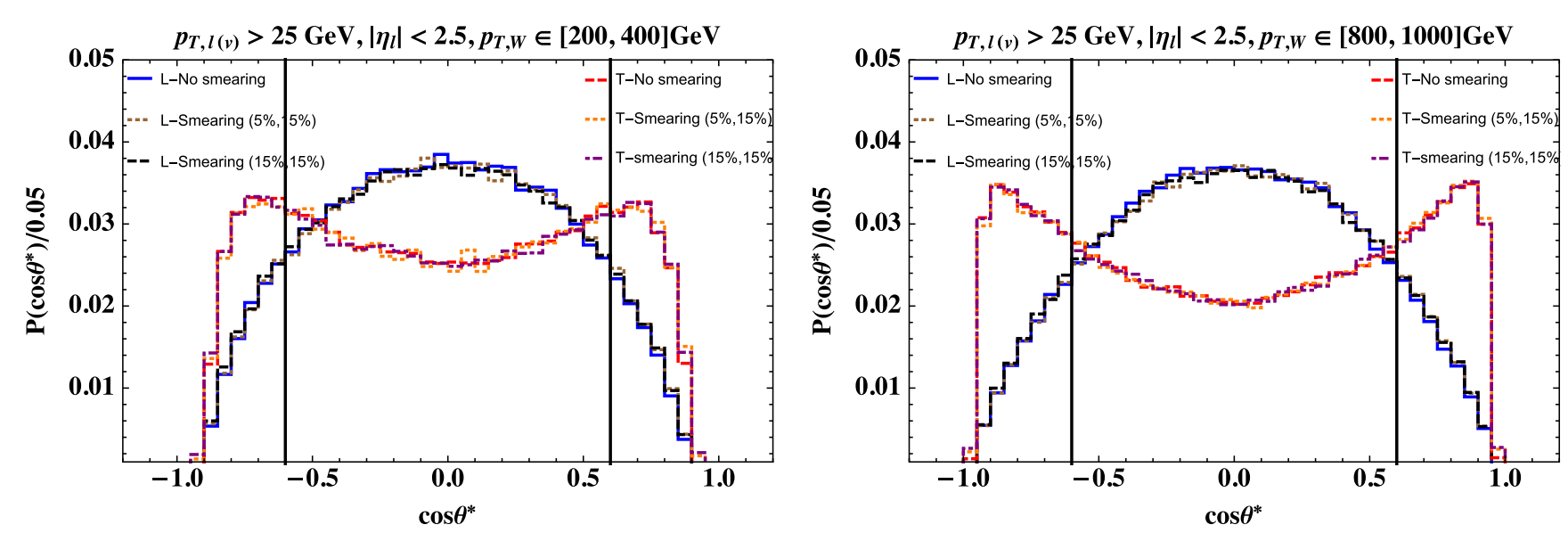

FIG. 2. Distribution of the $\cos \theta^{*}$ for the longitudinal $W$ and transverse $W$ bosons with $p_{T, W} \in[200,400]$ GeV (left plot) and $p_{T, W} \in[800,1000] \mathrm{GeV}$ (right plot). The transverse $W$ bosons include both + and - helicities. The distributions are normalized to 1 . The blue and the red lines are for the truth-level leptons and neutrinos, obtained from MadGraph5 [40] LO simulation. The brown and orange lines are obtained by smearing the truth-level energy of leptons by 5\% and neutrino by $15 \%$. The black and purple lines are obtained by smearing the truth-level energy of both leptons and neutrinos by $15 \%$.

Since the total cross section is dominated by the production of the transversely polarized $W$ 's, Eq. (12) explains the flat contours for this ratio in the right panel of Fig. 1 in the large $p_{T}$ regime. The factor $1 / 8$ in front of the right-hand side of Eq. (12) also explains the small value $\sim 0.1$ in the most central region with $\cos \theta_{V} \rightarrow 0$.

A very similar analysis applies to $W Z$ except that there is an amplitude zero for the transverse mode production in the central region. More specifically (again neglecting the contribution from the hypercharge), the squared amplitudes for the production of longitudinally and transversely polarized modes are

$$
\begin{aligned}
\left|\mathcal{M}_{W Z}^{T T}\right|^{2} & =\frac{g^{4}}{32}\left(\frac{s}{t}-\frac{s}{u}\right)^{2} \sin ^{2} \theta_{V}\left(1+\cos ^{2} \theta_{V}\right), \\
\left|\mathcal{M}_{W Z}^{L L}\right|^{2} & =\frac{g^{4}}{16} \sin ^{2} \theta_{V} .
\end{aligned}
$$

As in the case of $W W$ production, the squared amplitudes are the same for initial states $u \bar{d}+\bar{d} u$ and $d \bar{u}+\bar{u} d$. Equation (13) then explains the flat behavior for the contours of the ratio in the left panel of Fig. 1. In contrast to the $W W$ channel, here the transverse amplitude vanishes in the $\cos \theta_{V} \sim 0$. The ratio of the polarized production cross section is

$$
\frac{d^{2} \sigma_{W Z}^{L L}}{d^{2} \sigma_{W Z}^{T T}} \sim \frac{1}{8 \cos ^{2} \theta_{V}} \frac{1-\cos ^{2} \theta_{V}}{1+\cos ^{2} \theta_{V}}
$$

where it is clear that the longitudinal component is dominant in the central region, also shown in Fig. 1. Since it can be challenging to fully distinguish hadronic $W$ and $Z$ at the LHC, both signal and background will receive contributions from both $W W$ and $W Z$ channels.
Therefore, event selection based on simple kinematical cuts such as $p_{T}^{V}$ and $\cos \theta_{V}$ will always suffer from the contamination from the transversely polarized $W$ 's and may not achieve optimal results.

For the semileptonic channel, polarization tagging using the information of the decay products can provide additional information to further enhance the signal. Such a strategy has been considered in Ref. [36]. Here, we further explore its use in the case under consideration. The basic strategy is based on the well-known results that the distribution of the polar angle $\theta^{*}$ for the lepton in the $W$-rest frame are different for the longitudinally and transversely polarized $W$ bosons. The $z$-axis is typically chosen as the direction of the momentum of the $W$ boson in the laboratory frame [36]. The probability distributions of the polar angle for different helicity states in the $W^{+}$decay are given by (see Appendix B)

$P_{+}=\frac{3}{8}\left(1-\cos \theta^{*}\right)^{2}, \quad P_{-}=\frac{3}{8}\left(1+\cos \theta^{*}\right)^{2}, \quad P_{0}=\frac{3}{4} \sin ^{2} \theta^{*}$.

Note that $\cos \theta^{*}$ can be obtained directly from the momenta of the lepton and neutrino in the laboratory frame $\mathrm{as}^{3}$ (see Appendix B for a more detailed derivation)

$$
\cos \theta^{*}=\frac{E_{\ell}-E_{\nu}}{\left|\vec{p}_{\ell}+\vec{p}_{\nu}\right|}
$$

Normalized distributions of reconstructed $\cos \theta^{*}$ from longitudinally and transversely polarized $W$ 's are shown in

\footnotetext{
${ }^{3}$ In practice, the transverse momentum of the neutrino is identified with the missing energy. The longitudinal momentum of the neutrino is obtained by imposing the mass shell conditions for the neutrino and the $W$ boson.
} 
TABLE III. Two benchmarks for the longitudinal and transverse polarization tagging. The transverse $W$ bosons include both + and - helicities with equal probability.

\begin{tabular}{lcccc}
\hline \hline Cut & $\left|\eta_{W, Z}\right|<2.5$ & $p_{T, \ell(\nu)}>25 \mathrm{GeV},\left|\eta_{\ell}\right|<2.5$ & $\left|\cos \theta^{*}\right|<0.6$ & $\epsilon_{p_{T}, \eta} \times \epsilon_{\cos \theta^{*}}$ \\
\hline & & $p_{T, W} \in[200,400] \mathrm{GeV}$ & \\
Efficiency L & 0.572 & 0.943 & 0.810 & 0.764 \\
Efficiency T & 0.572 & 0.776 & 0.665 & 0.516 \\
& & $p_{T, W} \in[800,1000] \mathrm{GeV}$ & & \\
Efficiency L & 0.853 & 0.995 & 0.791 & 0.787 \\
Efficiency T & 0.854 & 0.921 & 0.553 & 0.509 \\
\hline \hline
\end{tabular}

Fig. 2. ${ }^{4}$ A major uncertainty in reconstructing the rest frame of the $W$ boson is the detector resolution in measuring the momenta of its decay products. As an example, we can use the CMS detector performance during LHC Run 1 [42]. For the electrons with $p_{T} \sim 45 \mathrm{GeV}$, the energy resolution is better than $2 \%$ in the central region $(|\eta|<0.8)$ and is $2 \%-5 \%$ elsewhere. For the muons, the energy resolution is $1.3 \%-$ $2.0 \%$ in the barrel and better than $6 \%$ in the end caps in the $p_{T}$ region of $[20,100] \mathrm{GeV}$. For the high $p_{T}$ muons, the resolution in the barrel is better than $10 \%$ up to $1 \mathrm{TeV}$. The jet energy resolution is approximately given by the following formula:

$$
\frac{\Delta E}{E} \approx \frac{100 \%}{\sqrt{E[\mathrm{GeV}]}} \oplus 5 \%
$$

Usually, the transverse missing energy resolution is dominated by the hadronic activity of the event. Similar results can be found for the ATLAS detector [43]. To estimate the resolution effects on the $\cos \theta^{*}$ distribution, we included the Gaussian smearing of the lepton and neutrino energy scale with the following two benchmark resolutions:

$$
\begin{array}{ll}
\text { (1) } \frac{\Delta E_{\ell}}{E_{\ell}}=5 \%, & \frac{\Delta E_{\nu}}{E_{\nu}}=15 \%, \\
\text { (2) } \frac{\Delta E_{\ell}}{E_{\ell}}=15 \%, & \frac{\Delta E_{\nu}}{E_{\nu}}=15 \% .
\end{array}
$$

From the plots in Fig. 2, we can see that the distribution is relatively stable under such smearing. This is due to the fact that $\cos \theta^{*}$ is reconstructed as a ratio, as shown in Eq. (16).

From Fig. 2 and Eq. (16), we see that the decay products are more central (forward) for longitudinally (transversely) polarized W's. This implies that the energies of the lepton and the neutrino in the lab frame tend to be symmetric for the longitudinally polarized $W$ boson. On the other hand, the decay products of $W$ 's with transverse polarization are more asymmetric. One of them tends to be hard, while the

\footnotetext{
${ }^{4}$ Note that for the quarks from hadronically decaying $W$ bosons, the distribution is the same. However, it is not possible to construct similar simple observables since we cannot identify the charge of the quark very well. Instead, some jet substructure variables need to be used to take advantage of this information.
}

other tends to be soft. Due to these kinematical differences, the $p_{T}, \eta$ cut on the charged leptons will already have some differential power on the longitudinal and transverse $W$ 's. In addition, we can impose a cut on the reconstructed $\cos \theta^{*}$ directly to further distinguish the two polarizations. In Table III, we have presented the effect of these cuts in two different kinematical regimes, one with moderately boosted $W$ boson $p_{T, W} \in[200,400] \mathrm{GeV}$, and the other with highly boosted $W$ boson $p_{T, W} \in[800,1000] \mathrm{GeV}$. Table III shows that $\cos \theta^{*}$ cuts can help with the signal significantly for a highly boosted region. For the moderately boosted region, $p_{T}, \eta$ cuts on leptons are already quite useful in suppressing the contamination from transverse $W$ 's. The addition of a cut on $\cos \theta^{*}$ does not significantly improve it. Based on this discussion, in the following analysis, we will use the following values for the polarization tagging ${ }^{5}$ :

$\epsilon_{L} \equiv \epsilon_{p_{T}, \eta}^{L} \times \epsilon_{\cos \theta^{*}}^{L}=0.75, \quad \epsilon_{T} \equiv \epsilon_{p_{T}, \eta}^{T} \times \epsilon_{\cos \theta^{*}}^{T}=0.5$.

The difference in the distribution of decay products also has a direct impact on tagging the hadronically decaying $W$ boson using the jet substructure method, with the longitudinal $W$-tagging efficiency higher by $40 \%$ (see Ref. [46]). One can also use jet substructure observables to develop a polarization tagger based on these kinematical features. We will leave this interesting topic for a future study. For this moment, we will assume the same polarization tagging efficiencies for the hadronically decaying $W$, $Z$ gauge bosons as Eq. (19). ${ }^{6}$

The dominant reducible background for the semileptonic channel is expected to be $W+$ jets, as shown in the $8 \mathrm{TeV}$

\footnotetext{
${ }^{5}$ The reconstruction efficiency of the electrons and the muons has mild dependence on the $\eta$, which ranges from $95 \%$ to $99 \%$ for $|\eta|<2.5[44,45]$. In our estimate, we have neglected the small difference.

${ }^{6}$ For the reducible backgrounds, we have assumed the same polarization tagging efficiencies as Eq. (19). This is fine for the leptonically decaying $W$ boson in the $W+$ jets background because of its transverse nature. For the QCD jet faking the hadronically decaying $W, Z$ bosons, it remains to be seen to what extent some jet shape variable carrying information of $\cos \theta^{*} \operatorname{could}$ help. The assumption does not affect our second benchmark in Eq. (20), as we assume that the reducible backgrounds are negligible.
} 


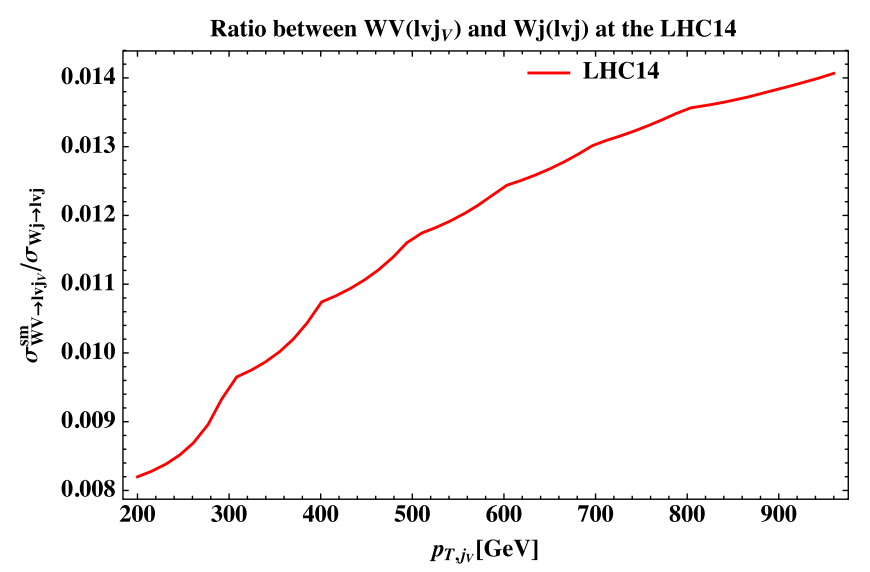

FIG. 3. The cross section ratio between SM $W V \rightarrow \ell \nu j_{V}$ and $W j \rightarrow \ell \nu j$ at the $14 \mathrm{TeV}$ LHC. The branching ratios to the particular final states are taken into account. We have required $\left|\eta_{W, j}\right|<2.5$.

analysis $[47,48]$. We show in Fig. 3 the LO cross section ratio between the SM $W V \rightarrow \ell \nu j_{V}$ and $W j \rightarrow \ell \nu j$ as a function of $p_{T}^{V}$ at the $14 \mathrm{TeV}$ LHC, where $j_{V}$ denotes the jet resulting from the hadronic decay of a vector boson. The simulation is carried out at the parton level using MadGraph [40], and we have required $\left|\eta_{V, j}\right|<2.5$. We see that this ratio ranges from $1 \%$ to $1.5 \%$ as $p_{T}^{\text {jet }}$ increases from $200 \mathrm{GeV}$ to $1 \mathrm{TeV}$. The most important tool to suppress this background is tagging hadronically decaying $W, Z$ using jet substructure observables. In Ref. [49], the ATLAS Collaboration studied the performance of the $W$-boson tagging in Run 2 and made projections of the efficiency of $W$-tagging and the rejection of the QCD-jet background. A benchmark point in the $p_{T}^{\text {jet }}$ range [500, $1000] \mathrm{GeV}$ for the $W$-tagging efficiency is $\epsilon_{W}^{\mathrm{tag}}=0.3$, while the miss-tagging efficiency for the QCD jet is $\epsilon_{j}^{\text {miss }}=0.004$. Combining this with the cross section ratio shown in Fig. 3, we could suppress the reducible background $W+$ jets to the same order of SM $W V$ production in the semileptonic channel. Reference [49] does not show the results for the $W$-tagging efficiency below 0.3 . In an earlier study of Ref. [50], the ATLAS Collaboration has shown the $W$-tagging efficiency below 0.3 , but with higher overall miss-tagging efficiency for the QCD jet. For the $\epsilon_{W}^{\mathrm{tag}}=0.3$, the miss-tagging rate is $\epsilon_{j}^{\mathrm{miss}}=0.006$, while for $\epsilon_{W}^{\mathrm{tag}}=0.1$, the miss-tagging rate is $\epsilon_{j}^{\text {miss }}=0.0014$. Compared with Ref. [50], the authors of Ref. [49] have improved the QCDjet miss-tagging rate by $33 \%$ for the $\epsilon_{W}^{\mathrm{tag}}=0.3$. If we assume the same improvement can be achieved for the case of $\epsilon_{W}^{\text {tag }}=0.1$, the miss-tagging rate for the QCD jet becomes $\epsilon_{j}^{\text {miss }}=0.0009$. The resulting reducible background for the $W V$ channel is roughly $20 \%$ of the SM $W V$ process and thus is subdominant. In our study, we will assume that for $\epsilon_{W}^{\operatorname{tag}}=0.1$, the reducible backgrounds can be reduced to a negligible level. We choose the following two benchmarks for the performance of vector boson tagging:

$$
\begin{aligned}
\epsilon_{V}^{\mathrm{tag}} & =0.3, & & n_{\text {red }}=n_{\text {irred }} \\
\epsilon_{V}^{\mathrm{tag}} & =0.1, & & n_{\text {red }}=0,
\end{aligned}
$$

where $V$ denotes the hadronically decaying $W, Z$ bosons. $n_{\text {irred }}$ is the number of irreducible background events, which come from SM $W V$ production. $n_{\text {red }}$ is the number of reducible background events which mostly come from SM $W+$ jets production. We have assumed that the tagging efficiencies for hadronically decaying $Z$ 's and $W$ 's are similar.

To summarize, the cross section in the semileptonically decaying channel from $W V$ production is given by

$$
\begin{aligned}
\sigma_{\text {semi-lep }}= & \sum_{p, p^{\prime}=L, T} \sigma_{W W}^{p p^{\prime}}\left(p_{T, W}>200 \mathrm{GeV},\left|\eta_{W}\right|<2.5\right) \times B R_{W W \rightarrow \ell \nu j j} \times \epsilon_{V}^{t a g} \times \epsilon^{p} \times \epsilon^{p^{\prime}} \\
& +\sum_{p, p^{\prime}=L, T} \sigma_{W Z}^{p p^{\prime}}\left(p_{T, V}>200 \mathrm{GeV},\left|\eta_{V}\right|<2.5\right) \times B R_{W Z \rightarrow \ell \nu j j} \times \epsilon_{V}^{\mathrm{tag}} \times \epsilon^{p} \times \epsilon^{p^{\prime}},
\end{aligned}
$$

with various efficiencies taking on benchmark values discussed in this section.

\section{B. $\boldsymbol{V} \boldsymbol{h}$ production}

For the $V h(b \bar{b})$ processes, the longitudinal component is dominant in the high-energy region for the SM. Therefore, we would not need to worry about contamination from final states with transverse polarization. In this case, suppressing the reducible background is essential to enhance the new physics effects. The dominant reducible backgrounds are
$V b \bar{b}, t \bar{t}$, and single top processes. It has been firmly established that the use of the jet substructure method can be effective in separating signal from background in the kinematical regime where the Higgs has a sizable boost $[17,29]$. This is also the regime where new physics effects considered here are enhanced. In particular, the authors of Ref. [17] studied the prospect for the discovery of the SMlike Higgs using a boosted Higgs tagging method, mainly in the $W h \rightarrow \ell \nu b \bar{b}$ channel. They demonstrated that, in the kinematic region $p_{T}^{V}>200 \mathrm{GeV}$, a signal to background 
ratio of $S_{\mathrm{SM}} / B_{\text {red }} \sim 0.2$ is achievable. Here, $S_{\mathrm{SM}}$ refers to the rate of SM $W h$-associated production, while $B_{\text {red }}$ is the rate of the reducible background. The signal efficiency obtained by the analysis using jet substructure in Ref. [17] depends on the $p_{T}$ bins. For the bins [200,400], [400,600], and $>600 \mathrm{GeV}$, the efficiencies are $0.1,0.2$, and 0.3 , respectively. ${ }^{7}$ More recently, the author of Ref. [18] studied these SM processes in the 0-, 1-, and 2-lepton states at the $13 \mathrm{TeV}$ LHC using a combination of boosted Higgs tagging variables. They obtained $S_{\mathrm{SM}} / B_{\text {red }} \sim 1$ with signal efficiency $\epsilon_{\text {tot }} \sim 0.1$ in the kinematic region $p_{T}^{V}>200 \mathrm{GeV}$. Of course, such phenomenological studies of the performance of the Higgs taggers and background rejection power are not fully realistic; they will need to be further studied by the experimental collaborations. At the same time, we also expect potential improvement both on the optimization of the variables and the reduction of experimental systematics. In our projection for the potential of HL-LHC, we will use the following benchmark:

$$
\epsilon_{\mathrm{tot}}=0.1, \quad n_{\text {red }}=n_{\text {irred }}
$$

in the 0-, 1-, and 2-lepton channels of $V h$ production, focusing on the boosted regions $p_{T, V}>200 \mathrm{GeV}$. Here, $n_{\text {irred }}$ refers to the number of events from the SM $V h$ production.

\section{Reach of the scale of new physics}

Based on our analysis of the semileptonic channels of diboson production, we now turn to the reach of new physics, parametrized by the dimension- 6 EFT operators in Eq. (2), through precision measurement in these channels. We make projections for the $95 \%$ confidence level reach of the scale $\Lambda$, denoted as $\Lambda_{95 \%}$, while setting the corresponding Wilson coefficient $c_{i}=1$.

As shown in Table II, production of diboson final states in the high-energy limit only depends on a certain combination of the EFT operators. Hence, in generating signal events, it is sufficient to include one of the operators in the combination. In particular, we generate the events using the $\mathcal{O}_{H W}$ operator for the combination $c_{q_{L}}^{(3)}$, while for the combination $c_{B}+c_{H B}-c_{2 B}$, we use operator $\mathcal{O}_{H B}$. We are not going to discuss the $U(1)_{Y}$ current-current fermionic operators $\left(\mathcal{O}_{R}^{u}, \mathcal{O}_{R}^{d}, \mathcal{O}_{L}^{q}\right)$, as the sensitivity to them is expected to be similar to that of $\mathcal{O}_{H B}$. We "turn on" one operator at a time. Including multiple operators at the same time can lead to potential correlations and flat directions. We will leave a more comprehensive treatment for a future study. We first show the bound from $W V, W h$ channels in each diboson invariant mass (or equivalently parton

\footnotetext{
${ }^{7}$ The number of events in each bin is given by $n^{i}=\sigma \times \mathrm{BR} \times \epsilon_{\mathrm{tot}}^{i}$.
}

center-of-mass energy) bin in Fig. 4 for integrated luminosities $L=300 \mathrm{fb}^{-1}$ or $L=3 \mathrm{ab}^{-1}$. For the studies of the semileptonically decaying channel of $W V$ by CMS at $8 \mathrm{TeV}$ [47], the systematics is dominated by the $W+$ jets background normalization, which is around 20\%. We expect that significant improvement in the HL-LHC, and the systematics can be reduced. Similar expectations apply to the $V h$ channels. In making this figure, we have assumed that the systematic error is $5 \%$. For our final combined results presented later, we vary the systematics between $3 \%$ and $10 \%$.

For the $W V$ channel, in each diboson invariant mass bin, we divided the partonic scattering angle $\cos \theta_{V}$ into four bins: $[0,0.2],[0.2,0.4],[0.4,0.6]$, and $[0.6,1.0]$. Then, we combined the bins with a number of events greater than 5 . This effectively put a cut on $\cos \theta_{V}$ which enhances the longitudinal new physics signal. From Fig. 4, we can see that higher energy bins, or equivalently larger $m_{W V}$ or $m_{W h}$ bins, generically yield better reaches. This is due to the inclusion of the systematic error, which limits the effectiveness of lower energy bins. For the high-luminosity LHC ( $L=3 \mathrm{ab}^{-1}$ ), the reach of the cutoff $\Lambda_{95 \%}$ in each diboson invariant mass bin is larger than the value of $m_{W V}\left(m_{W h}\right)$. Therefore, the reach is consistent with effective field assumptions from integrating out weakly coupled UV physics with $c_{q_{L}}^{(3)} \sim 1$. On the other hand, for integrated luminosity $L=300 \mathrm{fb}^{-1}$, not all the bins can be used to put a consistent bound for the $\Lambda$ in the weakly coupled theory $[12,51]$. It is still useful when the new physics is strongly coupled and the Wilson coefficients are enhanced by the strong coupling, as will be discussed in Sec. IV. In Fig 4, we have also plotted the limit on the validity of EFT in the most strongly coupled case $c_{q_{L}}^{(3)} \sim \frac{(4 \pi)^{2}}{g^{2}}$ (orange dashed line), which can arise if $q_{L}$ is fully composite.

We have also evaluated the reach on $\mathcal{O}_{3 W}$ using the semileptonically decaying $W V$ channel. The result is shown in Fig. 5, where we have performed an analysis similar to the $\mathcal{O}_{H W}$ case, i.e., using similar $\cos \theta_{V}$ bins and an assumption about the reducible backgrounds. As expected, the sensitivity to the $\mathcal{O}_{3 W}$ operator is weaker than the $\mathcal{O}_{H W}$ operator. This is due to the fact that the new signal from the $\mathcal{O}_{3 W}$ operator does not interfere with SM amplitudes (see Table II and also Ref. [33]). In fact, it only contributes to diboson states with helicities.$\pm \pm{ }^{8}$ The corresponding SM amplitudes with the same helicities go to zero in the high-energy limit, scaling like $m_{W}^{2} / E^{2}$. From Fig. 5, we can infer that the reach is in mild tension with the weakly coupled effective field theory even for the

\footnotetext{
${ }^{8}$ In principle, we can use the correlation between the $\cos \theta^{*}$ distribution of the two gauge bosons to distinguish the ++ and +- helicity final states. But for the semileptonically decaying channel, since we cannot distinguish the up-type quark from the down-type quark for the $W$-boson decay, the information will be lost.
} 

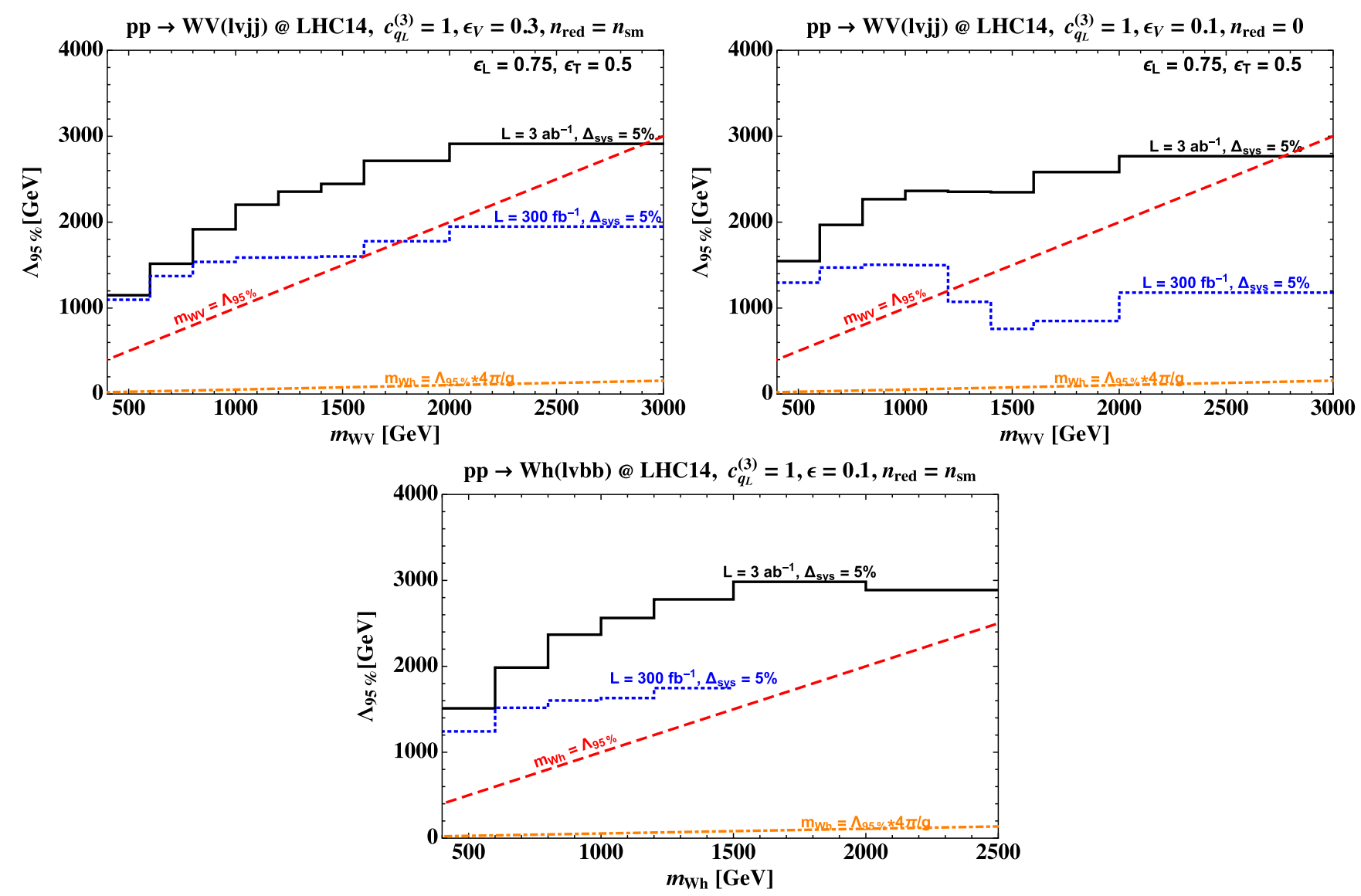

FIG. 4. $\Lambda_{95 \%}$, the $95 \%$ lower limit on the scale $\Lambda$ at the LHC is shown as a function of parton center-of-mass energy $m_{W V}$. The Wilson coefficient is set to be $c_{q_{L}}^{(3)}=1$, and the limit is set using channels $p p \rightarrow W V \rightarrow \ell \nu q \bar{q}$ (upper two plots) and $p p \rightarrow W h \rightarrow \ell \nu b \bar{b}$ (lower plot) for integrated luminosities $L=300 \mathrm{fb}^{-1}$ (solid blue) and $L=3 \mathrm{ab}^{-1}$ (solid black). The dashed red line, for $m_{W V}=\Lambda_{95 \%}$, is the condition for the consistency of weakly coupled effective field theory. The dashed orange line, for $m_{W V}=\frac{4 \pi}{g} \Lambda_{95 \%}$, is the condition for the consistency of the most strongly coupled effective field theory [operator enhanced by $(4 \pi)^{2} / g^{2}$ ]. If the limit $\Lambda_{95 \%}>m_{W V}$ in a particular $m_{W V}$ bin, it is consistent with SM effective field theory. For the $W V$ process, we have explored two benchmark values for the boosted $V$-jet tagging efficiency and the reducible background, i.e., $\epsilon_{V}=0.3, n_{\text {red }}=n_{\mathrm{SM}}$ (upper left) and $\epsilon_{V}=0.1, n_{\text {red }}=0$ (upper right). In addition, we assume that the $W(V)$ polarization tagging efficiencies are $\epsilon_{L}=0.75, \epsilon_{T}=0.5$.

high-luminosity LHC. But for strongly coupled transverse gauge bosons, the Wilson coefficient can be enhanced by the strong coupling. In this case, the projected reach is consistent with effective field theory as long as the coupling is large enough (see the orange dashed line in Fig. 5 for the most strongly coupled case with $c_{3 W} \sim 4 \pi / g$ ). This reach may be further improved by using an azimuthal angle distribution of the decay information of the $W, Z$ bosons, which results in interference with the leading nonvanishing SM amplitude (see Refs. [52,53]). We will not explore this possibility further here.

Finally, we combine all the bins and make projections on the reach of cutoff $\Lambda$ for different operators in different processes. The results are summarized in Fig. 6. We have varied the systematics from $3 \%$ to $10 \%$. For the semileptonically decaying $W V$ channel, we only show the benchmark values for $\epsilon_{V}^{\text {tag }}=0.3, n_{\text {red }}=n_{\text {irred }}$. For the second benchmark point of Eq. (20), there is no big difference except that the dependence on the systematic uncertainty is weaker. This is because of the assumption of zero reducible background. From Fig. 6, we can infer that for the case of $c_{q_{L}}^{(3)}=1$, the most important bound comes from both $W h(\ell \nu b \bar{b})$ and $W V(\ell \nu j j)$ channels. Taking $\Delta_{\text {sys }}=5 \%$ as a benchmark point, the reaches in these two channels are comparable, around 3.8(2.5) TeV in the $W V(\ell \nu j j)$ channel for integrated luminosities $L=3 \mathrm{ab}^{-1}\left(300 \mathrm{fb}^{-1}\right)$ and $4.0(2.3) \mathrm{TeV}$ for the $W h$ channel. Note that $c_{q_{L}}^{(3)}$ is the combination of the operators $c_{q_{L}}^{(3)}=c_{W}+c_{H W}-c_{2 W}+4 c_{L}^{(3) q}$. If we assume that there is no big cancellation in different Wilson coefficients, we can compare the reach from diboson processes with the bound from EWPT at the LEP and Higgs coupling measurement at the HL-LHC, even though the latter two depend on different combinations of operators (see Table II). The operator $\mathcal{O}_{W}$ will contribute to the $S$-parameter $[38,54]$. 

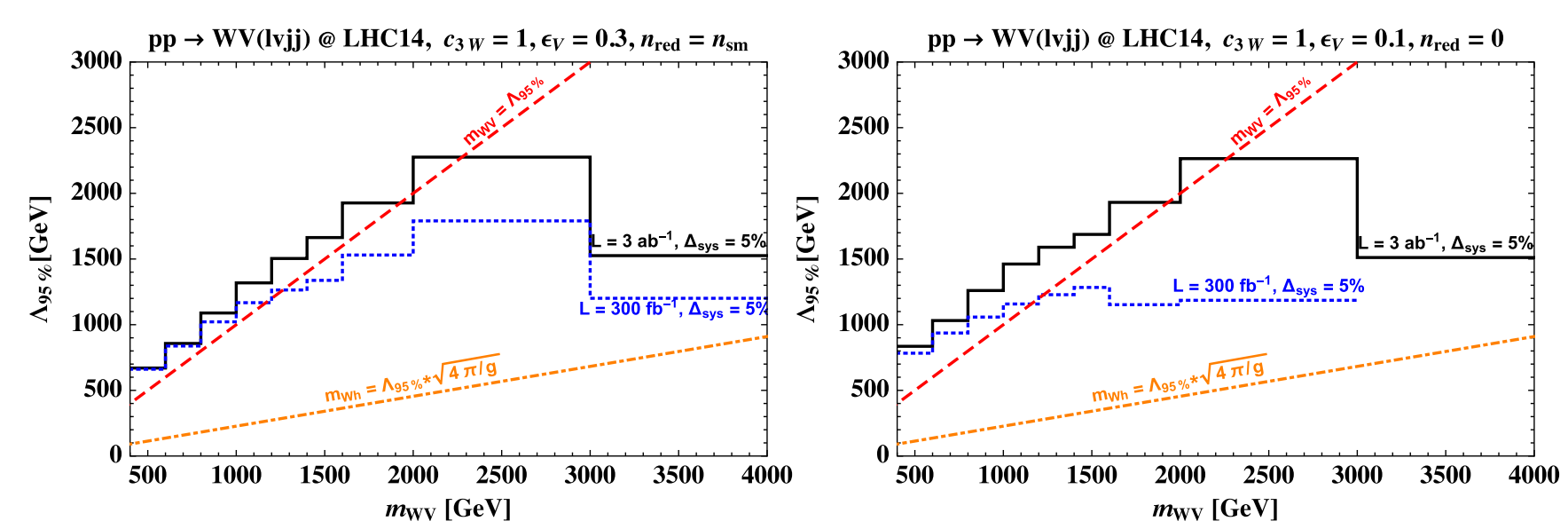

FIG. 5. $95 \%$ lower limit on the scale $\Lambda$ at the LHC for Wilson coefficient $c_{3 W}=1$ in the channel $p p \rightarrow W V \rightarrow \ell \nu q \bar{q}$, with systematics $\Delta_{\text {sys }}=5 \%$, for the integrated luminosities $L=300 \mathrm{fb}^{-1}$ (solid blue) and $L=3 \mathrm{ab}^{-1}$ (solid black). The dashed red line, for $m_{W V}=\Lambda_{95 \%}$, is the condition for the consistency of the weakly coupled effective field theory [the Wilson coefficient is $\mathcal{O}(1)$ ]. The dashed orange line, for $m_{W V}=\sqrt{\frac{4 \pi}{g}} \Lambda_{95 \%}$, is the condition for the consistency of the most strongly coupled effective field theory (the Wilson coefficient is $4 \pi / g$ ).

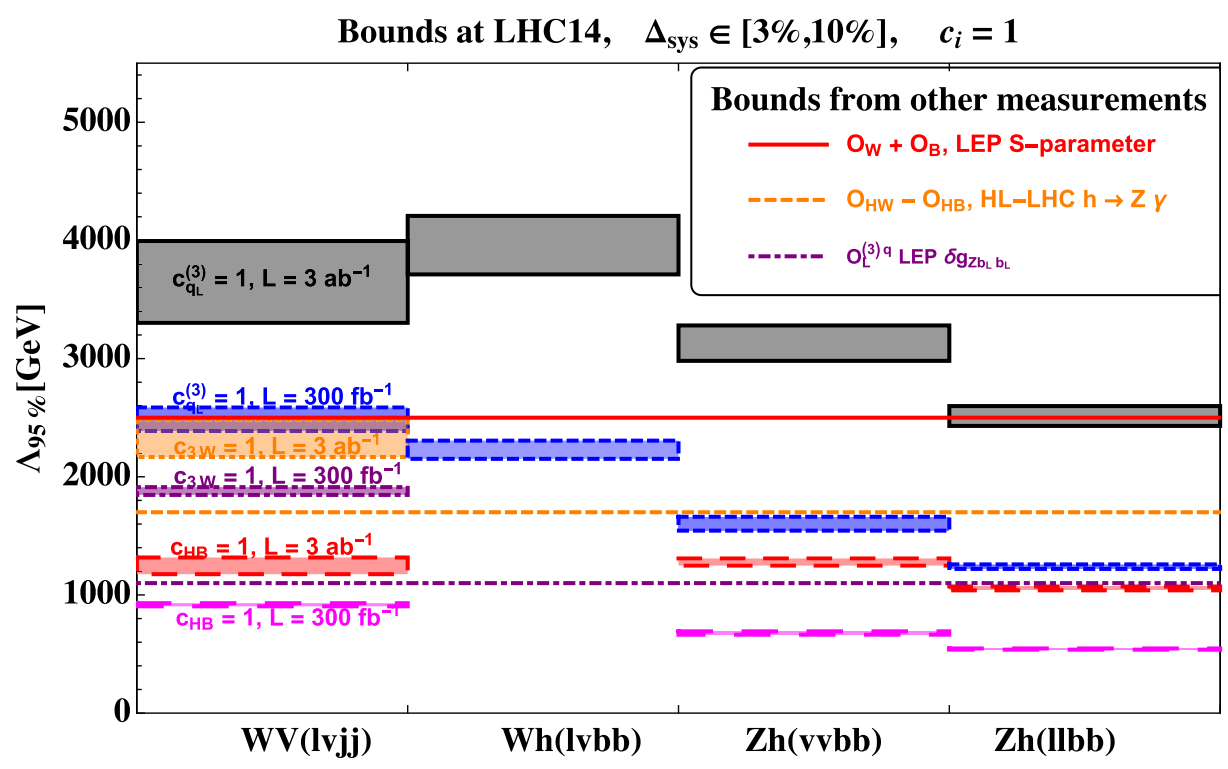

FIG. 6. Reach in different channels at the $14 \mathrm{TeV}$ LHC for different combinations of the operators assuming the systematic error varies from $3 \%$ to $10 \%$. The grey and blue regions denote the reach of the scale in the case of $c_{q_{L}}^{(3)}=1$ for integrated luminosities $L=3$ ab and $L=300 \mathrm{fb}^{-1}$, respectively. The red and magenta regions denote the reach in the case of $c_{B}+c_{H B}-c_{2 B}=1$ for integrated luminosities $L=3 \mathrm{ab}^{-1}$ and $L=300 \mathrm{fb}^{-1}$. The orange and purple regions denote the reach of the size of $\mathcal{O}_{3 W}$ operator with $c_{3 W}=1$, for integrated luminosities $L=3 \mathrm{ab}^{-1}$ and $L=300 \mathrm{fb}^{-1}$. We also show the present bound from the LEP S-parameter on the combination of operators $\mathcal{O}_{W}$ and $\mathcal{O}_{B}$ with $c_{W}+c_{B}=1$ (red dashed line), the bound from the $\operatorname{LEP} \delta g_{Z b_{L} \bar{b}_{L}}$ measurement on the operator $c_{L}^{(3) q}=1 / 4$ (purple dashed line), based on flavor-universal effects. The case of $c_{H W}-c_{H B}=1$ is shown as the orange dashed line from the $3 \mathrm{ab}^{-1}$ HL-LHC measurement of the $h \rightarrow Z \gamma$ decay partial width, with a projected precision of $\sim 20 \%$ from Ref. [23].

Suppose it is the dominant contribution; the bound is $\sim 2.5 \mathrm{TeV}$ at $95 \%$ C.L. for $c_{W}=1 . \mathcal{O}_{H W}$ will contribute to the Higgs rare process $h \rightarrow Z \gamma$. The $h \rightarrow Z \gamma$ measurement at HL-LHC will put a limit around $1.7 \mathrm{TeV}$ [23] for $c_{H W}=1$. For the flavor-universal operator $\mathcal{O}_{L}^{(3) q}$, from the $\mathrm{LEP} \delta g_{Z b_{L} b_{L}}$ measurement, the bound is around $1.1 \mathrm{TeV}$ for
$c_{L}^{(3) q}=1 / 4[55,56]{ }^{9}$ We have shown the three bounds as the red, orange, and purple dashed lines in Fig. 6. The comparison above shows that diboson measurement is very

\footnotetext{
${ }^{9} c_{L}^{(3) q}=1 / 4$ is chosen such that $c_{q_{L}}^{(3)}=1$ [see Eq. (3)].
} 
promising to probe the new physics scenario in which the operators considered here give the most important effect. For the operator $\mathcal{O}_{2 W}$, it will contribute to the four fermion operator by the equation of motion, especially to the DrellYan processes $q \bar{q} \rightarrow \ell^{+} \ell^{-}$. This has been studied in Ref. [57] and the expected reach is $13.4 \mathrm{TeV}$ at the HLLHC for $c_{2 W}=1$. Usually, this operator will be suppressed by a factor of $g^{2} / g_{*}^{2}$. However, in a certain scenario with strong multipole interactions (the so-called Remedios scenario) in Ref. [37], this operator may become as relevant as others. We will discuss this in detail in the next section. For the operator combinations of $c_{B}+c_{H B}-c_{2 B}$, the reach is relatively weak (1.3 TeV at the HL-LHC) from the diboson process. This is a result of the smallness of the hypercharge coupling $g^{\prime}$. This makes it difficult to compete with the S-parameter and $h Z \gamma$ measurement, and the reach is also not consistent with the weakly coupled effective field theory. We finally mention that the bound for $\mathcal{O}_{3 W}$ is 2.4 (1.9) $\mathrm{TeV}$ at $3 \mathrm{ab}^{-1}\left(300 \mathrm{fb}^{-1}\right)$, which is also only meaningful if its Wilson coefficient is enhanced by a strong coupling.

\section{REACH OF NEW PHYSICS SCALES IN DIFFERENT SCENARIOS}

In Sec. III, we have presented the projection on the reach of $\Lambda$ in a model independent way with unit Wilson coefficients $\left(c_{q_{L}}^{(3)}=1, c_{3 W}=1\right.$, etc.). In different new physics scenarios, the size of Wilson coefficients can be quite different. Assuming that the new physics is broadly characterized by a mass scale of new states $m_{*}$ and a coupling $g_{*}$, the Wilson coefficients are

$$
c_{i} \sim \frac{g_{*}^{n}}{g_{\mathrm{SM}}^{n}}\left(\frac{g_{*}^{2}}{16 \pi^{2}}\right)^{n_{\mathrm{Loop}}}, \quad n \leq n\left(\mathcal{O}_{i}\right)-2,
$$

where $g_{\mathrm{SM}}$ denotes the SM gauge and Yukawa couplings $g$, $g^{\prime}, y_{f} . n\left(\mathcal{O}_{i}\right)$ is the number of fields in the operator $\mathcal{O}_{i}$. In particular, $n\left(\mathcal{O}_{W, B, H W, H B, 3 W}\right)=3, \quad n\left(\mathcal{O}_{2 W, 2 B}\right)=2$, and $n\left(\mathcal{O}_{L, R}^{q}\right)=4$. Note that a covariant derivative is not counted as a field. $n_{\text {Loop }}$ is the number of loops needed to generate the operator. Note that $n$ can also be negative. The bounds $\Lambda_{95 \%}$ obtained in the previous section can be easily translated into the bounds on the mass scale $m_{*}$, as functions of Wilson coefficients $c_{i}$ :

$$
m_{*}>\Lambda_{95 \%}^{c_{i}=1} \times \sqrt{c_{i}} .
$$

In the following, we will consider the strongly interacting light Higgs (SILH), strong multipole interaction (Remedios), and the (partially) composite fermion scenarios.

\section{A. SILH scenario}

We start with the SILH scenario [58]. There are two basic assumptions. First, the Higgs and the longitudinal components of the SM gauge bosons are pseudoNambu-Goldstone bosons associated with the global symmetry breaking in a strongly interacting sector [59]. In addition, the SM fermions acquire masses from their linear mixing with corresponding strongly interacting sector states (the so-called partial compositeness [60]). This leads to the following power-counting rules for the Wilson coefficients:

(i) Each Higgs and Goldstone field will be associated with a strong coupling $g_{*}$ in the operators which preserve the global symmetries of the strongly interacting sector, including those which are nonlinearly realized.

(ii) Explicit breaking of the strongly interacting sector symmetries will be associated with SM gauge couplings and Yukawa couplings, $g, g^{\prime}$, and $y_{f}$.

Following these rules, we have summarized the size of the Wilson coefficients of the operators for the SILH scenario in the second row of Table IV. None of the operators considered in our paper is enhanced by the strong coupling $g_{*}$, mainly due to the fact that the transversely polarized gauge bosons belong to the elementary sector. In the second row of Table V, we summarize the reach of the mass scales in the SILH scenario from the HL-LHC measurements of the diboson, $h \rightarrow Z \gamma, h \rightarrow \gamma \gamma$ [23], and dilepton processes. For comparison, we have also included the bound from $S$-parameter measurement. In comparison with other measurements, diboson processes have the best reach in the SILH scenario.

\section{B. Strong multipole interaction (Remedios) scenario}

The authors of Ref. [37] considered the possibility that the SM transverse gauge bosons are part of the strong dynamics. This so-called Remedios scenario is based on the observation that the normal SM gauge interactions (monopole) and multipole interactions (involving the field strength and its derivatives) have different symmetry structures. Therefore, they can have different coupling strengths in principle. The small Standard Model couplings, such as $g$, control the renormalizable interactions between the gauge boson and the fermions. At the same time, the large coupling $g_{*}$ determines the strength of the multipole

TABLE IV. Power counting of the size of the Wilson coefficients in different scenarios, where $g_{*}$ denotes the coupling in the strong sector. For completeness, we have added $\mathcal{O}_{B B}=g^{2} H^{\dagger} H B_{\mu \nu} B^{\mu \nu}$.

\begin{tabular}{lccccccc}
\hline \hline Model & $\mathcal{O}_{2 W}$ & $\mathcal{O}_{2 B}$ & $\mathcal{O}_{3 W}$ & $\mathcal{O}_{H W}$ & $\mathcal{O}_{H B}$ & $\mathcal{O}_{W, B}$ & $\mathcal{O}_{B B}$ \\
\hline SILH & $\frac{g^{2}}{g_{*}^{2}}$ & $\frac{g^{2}}{g_{*}^{*}}$ & $\frac{g^{2}}{16 \pi^{2}}$ & $\frac{g_{*}^{2}}{16 \pi^{2}}$ & $\frac{g_{*}^{2}}{16 \pi^{2}}$ & 1 & $\frac{g^{2}}{16 \pi^{2}}$ \\
Remedios & 1 & 1 & $\frac{g_{*}}{g}$ & & & & \\
Remedios + MCHM & 1 & 1 & $\frac{g_{*}}{g}$ & 1 & 1 & 1 & 1 \\
Remedios + ISO(4) & 1 & 1 & $\frac{g_{*}}{g}$ & $\frac{g_{*}}{g}$ & 1 & 1 & 1 \\
\hline \hline
\end{tabular}


TABLE V. The bounds (in TeV) for different scenarios from different measurements. The LHC measurements are prospectives at the integrated luminosity $L=3 \mathrm{ab}^{-1}$.

\begin{tabular}{lccccc}
\hline \hline Model & Diboson & S-parameter & LHC $h \rightarrow Z \gamma$ & LHC $h \rightarrow \gamma \gamma$ & LHC dilepton \\
\hline SILH & 4.0 & 2.5 & $1.7 \sqrt{\frac{g_{*}}{4 \pi}}$ & 0.34 & $0.69 \sqrt{\frac{4 \pi}{g_{*}}}$ \\
Remedios & $10.6 \sqrt{\frac{g_{*}}{4 \pi}}$ & & & & 13.4 \\
Remedios + MCHM & $10.6 \sqrt{\frac{g_{*}}{4 \pi}}$ & 2.5 & 1.7 & 6.5 & 13.4 \\
Remedios + ISO(4) & $17.6 \sqrt{\frac{g_{*}}{4 \pi}}$ & 2.5 & $7.5 \sqrt{\frac{g_{*}}{4 \pi}}$ & 6.5 & 13.4 \\
\hline \hline
\end{tabular}

interactions of the gauge bosons with the resonances of the strong sector. This will lead to the following new powercounting rules for the gauge bosons:

(i) The field strengths of the gauge boson and their derivatives are associated with a strong coupling $g_{*}$, if the interactions preserve the global symmetries of the strong sector. The normal gauge interactions are realized by changing the partial derivative to the covariant derivative: $\partial_{\mu} \rightarrow D_{\mu}=\partial_{\mu}-i g A_{\mu}$.

In this case, the $\mathcal{O}_{3 W}$ operator is enhanced by the strong coupling, while the $\mathcal{O}_{2 W, 2 B}$ operators have $\mathcal{O}(1)$ Wilson coefficients. The power counting of these operators considered in this scenario have been summarized in the third row of Table IV. We can consider further the scenarios in which both transverse gauge bosons and Higgs bosons are part of the strong dynamics. Depending on the symmetry of the strong sector, we have two benchmark scenarios:

(i) Remedios + MCHM: The symmetry breaking of the strong sector will be $S O(5) \times \widetilde{S U(2)} \times U(1)_{X} \rightarrow$ $S O(4) \times \widetilde{S U(2)} \times U(1)_{X}$, where another global symmetry $\widehat{S U(2)}$ is needed to stabilize the Higgs potential.

(ii) Remedios + ISO(4): The symmetry breaking of the strong sector will be $\operatorname{ISO}(4) \times U(1)_{X} \rightarrow S O(4) \times$ $U(1)_{X}$, where the $\operatorname{ISO}(4)$ is the noncompact group $S O(4) \rtimes T^{4}$.

The corresponding power-counting rules for the size of the Wilson coefficients are presented in the fourth and fifth rows of Table IV. We summarize the reaches for these three benchmark scenarios from different measurements of Table V. Several comments are in order. If only the field strengths are strongly coupled (third row), the most relevant operators are $\mathcal{O}_{2 W}$ with $\mathcal{O}(1)$ Wilson coefficients and $\mathcal{O}_{3 W}$ with the enhanced Wilson coefficient $\sim \mathcal{O}\left(g_{*} / g\right)$. Dilepton measurements at HL-LHC will reach 13.4 TeV. The reach from diboson measurements is weaker, which is $10.6 \mathrm{TeV}$ for the most strongly interacting case $g_{*}=4 \pi$. The projection is similar for the Remedios Remedios + MCHM scenario. For the Remedios + ISO(4) scenario, $\mathcal{O}_{H W}$ is enhanced by the strong coupling $g_{*}$. Its Wilson coefficient is $g_{*} / g$. As a result, diboson measurement can reach higher $\sim 17.6 \sqrt{g_{*} / 4 \pi} \mathrm{TeV}$, which becomes better than dilepton measurement for large coupling $g_{*} \gtrsim 7$.

\section{Partially composite fermions}

Finally, we discuss the fermionic operators. We focus on the operators $\mathcal{O}_{L}^{(3) q}$, and we expect the conclusions for other fermionic operators are similar. We will also focus on the flavor-universal effects that are invariant under $S U(3)$ flavor transformation. Other effects will be suppressed by the Yukawa couplings under the assumption of minimal flavor violation (MFV) [61]. As discussed before, the LHC diboson measurement $\left(\Lambda_{95 \%}^{c_{L}^{(3) q}=\frac{1}{4}} \sim 4 \mathrm{TeV}\right)$ will be much better than the LEP measurement $\left(\Lambda_{95 \%}^{c_{L}^{(3) q}=\frac{1}{4}} \sim 1.1 \mathrm{TeV}\right)$ for such effects. Now if we assume that the SM fermions have some degrees of compositeness $\epsilon_{q_{L}}$ (for example, the partial compositeness scenario in Ref. [60]), the size of the Wilson coefficient of the $\mathcal{O}_{L}^{(3) q}$ by power counting is

$$
c_{L}^{(3) q_{L}} \sim \frac{1}{4} \frac{g_{*}^{2}}{g^{2}} \epsilon_{q_{L}}^{2},
$$

where we have factored out a $1 / 4$ factor to be consistent with the above consideration. The HL-LHC diboson measurement will reach the mass scale:

$$
m_{*} \gtrsim 77 \frac{g_{*}}{4 \pi} \epsilon_{q_{L}} \mathrm{TeV} \quad @ 95 \% \text { C.L. }
$$

In the meantime, the following four-fermion operator will also be present in the low-energy effective field theory:

$$
\mathcal{L}_{4 f}=\frac{g_{*}^{2} \epsilon_{q_{L}}^{4}}{m_{*}^{2}} \bar{q}_{L} \gamma^{\mu} q_{L} \bar{q}_{L} \gamma_{\mu} q_{L}
$$

This will lead to energy growing behavior in the dijet processes at the LHC. The present bound from ATLAS dijet measurement [62] at $13 \mathrm{TeV}$ with the integrated luminosity of $15.7 \mathrm{fb}^{-1}$ is given by (see Refs. [63,64])

$$
m_{*} \gtrsim 62 \frac{g_{*}}{4 \pi}\left(\epsilon_{q_{L}}\right)^{2} \mathrm{TeV} \quad \text { @ 95\%C.L. }
$$

The authors of Ref. [65] have studied the prospectives on the following operator at the HL-LHC with $13 \mathrm{TeV}$ centerof-mass energy ${ }^{10}$ :

\footnotetext{
${ }^{10}$ Actually, this operator arises from $-\frac{1}{2}\left(D_{\mu} G^{A \mu \nu}\right)^{2}$ by the equation of motion of the gluon fields.
} 


$$
-\frac{g_{*}^{2} \epsilon_{q}^{4}}{2 m_{*}^{2}}\left(\sum_{q} \bar{q} \gamma^{\mu} T^{A} q\right)^{2}
$$

where $T^{A}$ is the generators of the QCD $S U(3)_{c}$ group. The expected $95 \%$ C.L. bound on the scale is

$$
m_{*} \gtrsim 83 \frac{g_{*}}{4 \pi}\left(\epsilon_{q_{L}}\right)^{2} \mathrm{TeV} \quad @ 95 \% \text { C.L. }
$$

Although this operator is different from the one in Eq. (27), it can provide a rough idea about what scale can be probed in the diet process at the HL-LHC. We can see that for the smaller values of $\epsilon_{q}<0.9$, the LHC diboson measurement can be more promising than the dijet process.

\section{CONCLUSIONS}

The future runs of the LHC in the next decade or so will collect nearly 30 times more data than currently available. There is great potential to improve the precision measurements with this new data set. The measurements of the SM electroweak sector are particularly important, as it is closely related to new physics associated with electroweak symmetry breaking. Studies of diboson channels, $V V$ and $V h$, where $V$ can be the SM $W$ and $Z$, give a promising window into such new physics. Such measurements can be complementary to the direct search for new physics particles. In certain scenarios, new physics particles can be too heavy to be produced at the LHC. At the same time, their presence can lead to observable effects in precision measurements.

In this paper, we parametrize the new physics effects with dimension-6 EFT operators. We focus on operators which are most relevant for the diboson final states. In particular, we study the reach in the semileptonic final states. In order to fully take advantage of the larger effect of EFT operators at higher energies, we need to select final states which interfere with the SM background. While this is guaranteed for the $V h$ channel, we have to select longitudinally polarized $W$ and $Z$ in the $W W$ and $W Z$ channels. There are two possible strategies to achieve polarization tagging. First, the angular distributions of longitudinally and transversely polarized gauge bosons are different. This effect is most dramatic in the $W Z$ final state with the so-called amplitude zero in the central region for the transverse vector bosons. This has been crucial for the analysis in the pure leptonic channel [15]. For the semileptonic channel we studied here, since we cannot distinguish hadronic $W$ and $Z$ very well, this effect is less prominent. Another approach is to directly tag the polarization of the gauge boson by the angular distribution of their decay products. We find that this approach increased the sensitivity a lot in our analysis. For example, for the systematic $\Delta_{\text {sys }}=5 \%$, the HL-LHC reach on the mass scale of the operator combination $c_{q_{L}}^{(3)}=1$ has increased from 3.4 to $3.8 \mathrm{TeV}$ for benchmark $\epsilon_{V}^{\text {tag }}=0.3, n_{\text {red }}=n_{\text {irred }}$ and from 3.5 to $3.8 \mathrm{TeV}$ for the benchmark $\epsilon_{V}^{\mathrm{tag}}=0.1$, $n_{\text {red }}=0$. In our study, we use a combination of both approaches. Since the precision measurements typically focus on cases where $S / B$ is small, the sensitivity depends crucially on systematic error and background estimates (in particular, reducible background). For the reducible background of the semileptonic $W V$ channel, we have considered the dominant background $W+$ jets at the parton level and applied the $W$-tagging efficiency and QCD-jet misstagging efficiency based on the study of Ref. [49]. The resulting two benchmarks are summarized in Eq. (20). For the $V h(b \bar{b})$ channel, we have adopted the study of Ref. [18] about the reducible backgrounds in the 0-, 1-, and 2-lepton channels, which leads to Eq. (22) as our benchmark in these channels. Our results show that precision measurement at the LHC can have good sensitivity in probing new physics at the multiple-TeV scale. It can surpass the sensitivity of LEP precision measurements, such as those from the S-parameter and $Z$ coupling measurements. Compared with fully leptonic decaying $W Z$ channels, the semileptonic decay $W V$ channel has an order-of-magnitude larger rate. At the same time, semileptonic decay channels suffer from large reducible backgrounds. During the upcoming runs of the LHC, we expect significant improvement in understanding the reducible background and reducing systematics. Anticipating this, we make optimistic projections of the reducible background for the semileptonic decay channels based on extrapolations of the ATLAS study. Based on this, our result $\left(\Lambda_{95 \%}^{c_{q L}^{(3)}} \sim 4 \mathrm{TeV}\right)$ is better than the fully leptonic $W Z$ channel $\left(\Lambda_{95 \%}^{c_{L}^{(3)}} \sim 3.2 \mathrm{TeV}\right)$ studied in Ref. [15]. As an application of our result, we derived the reach of the new physics scale in several new physics scenarios. In the SILH scenario, which models the generic feature of composite Higgs models, the diboson measurement can be more sensitive than other experimental observables. For the scenario with strong multiple interactions (the so-called Remedios), the diboson is either slightly weaker or comparable with the measurements in the dilepton channel.

It is worth emphasizing that the estimates we made here are based on our assumptions about systematics and efficiencies achievable at the HL-LHC. More detailed and realistic studies, presumably based on real data and full-fledged simulations, would be necessary to determine the precise reach. In this sense, the numbers presented here are better considered as benchmarks or targets, which could give us good reach in these channels. We have also identified several directions in which improvements can be crucial to enhance the sensitivity in the diboson channel. Obviously, any new technique to tag the polarization of the vector bosons can be very helpful. A major direction to pursue is the tagging of polarization of the hadronic $W$ and $Z$. In addition, distinguishing hadronic $W$ and $Z$ can also be very helpful in enhancing longitudinal final states. 


\section{ACKNOWLEDGMENTS}

We are grateful to Andrea Tesi for numerous helpful discussions and collaboration during the early stages of this work. We would also like to thank Francesco Riva and Andrea Wulzer for helpful discussions and Nurfikri Norjoharuddeen, Oliver Majersky, and Ece Akilli for bringing Ref. [50] to our attention. L. T. W. is supported by DOE Grant No. DE-SC0013642. D. L. is supported in part by the U.S. Department of Energy under Contract No. DE-AC02-06CH11357.

\section{APPENDIX A: CROSS SECTIONS OF THE DIBOSON PROCESSES AT THE LHC}

In Table VI, we have reported the cross section for the diboson processes at the $14 \mathrm{TeV}$ LHC as a function of the cutoff $\Lambda$ in each $p_{T}$ bin with the Wilson coefficient $c_{H W}$ set to 1 . The cross sections are calculated using MadGraph [40] at LO simulation with parton level cuts $\left|\eta_{W, Z, h}\right|<2.5$. We can see clearly that the new physics effects manifest in the purely longitudinal helicity final states of $W, Z$ gauge bosons and the Higgs boson with energy growing behavior. It results in the fact that the coefficients of $1 / \Lambda^{2}$ become larger as the $p_{T}$ increases. In addition, the ratios between the coefficients of $1 / \Lambda^{4}$ and that of $1 / \Lambda^{2}$ grow as the $p_{T}$ becomes larger, which indicates that the larger value of $\Lambda$ is needed for $1 / \Lambda^{2}$ terms to dominate. For the processes including one transverse gauge boson and one longitudinal gauge boson (including the Higgs boson), the cross sections are comparable to the transverse one in the low $p_{T}$ bin $[0,400] \mathrm{GeV}$ and decrease very fast as $p_{T}$ increases. It results in less than $5 \%$ of the $L L$ one for the $W W, W Z$ processes and less than $1 \%$ of $V_{L} h$ for the $V h$ processes for the largest $p_{T}$ bin. For the SM $W W$, the purely transverse helicity final states $T T$ dominate over $L L$ by a factor of 16 in the moderately boosted region $p_{T} \in[200,400] \mathrm{GeV}$ and a factor of 12 in the highly boosted region $p_{T} \in[1000,1500] \mathrm{GeV}$. While for the $W Z$ process, the $T T$ cross section is only a factor of 3 of the $L L$ one in the moderately boosted region and becomes comparable to $L L$ in the highly boosted bin. This is due to the amplitude zero in this process as discussed in the main text.

TABLE VI. Helicity cross sections (in fb) for the diboson processes at the $14 \mathrm{TeV}$ LHC as a function of the cutoff $\Lambda$ (in TeV) in each $p_{T}$ bin with the Wilson coefficient $c_{H W}$ set to 1 .

\begin{tabular}{|c|c|c|c|}
\hline$\sigma(\mathrm{fb}), p_{T}(\mathrm{GeV})$ & {$[0,200]$} & {$[200,400]$} & {$[400,600]$} \\
\hline$W_{L}^{ \pm} Z_{L}$ & $784\left(1+\frac{0.116}{\Lambda^{2}}+\frac{0.00625}{\Lambda^{4}}\right)$ & $58.5\left(1+\frac{0.682}{\Lambda^{2}}+\frac{0.141}{\Lambda^{4}}\right)$ & $4.84\left(1+\frac{2.09}{\Lambda^{2}}+\frac{1.24}{\Lambda^{4}}\right)$ \\
\hline$W_{L(T)}^{ \pm} Z_{T(L)}$ & $1614\left(1+\frac{0.0610}{\Lambda^{2}}+\frac{0.00181}{\Lambda^{4}}\right)$ & $23.0\left(1+\frac{0.419}{\Lambda^{2}}+\frac{0.0611}{\Lambda^{4}}\right)$ & $0.598\left(1+\frac{1.40}{\Lambda^{2}}+\frac{0.623}{\Lambda^{4}}\right)$ \\
\hline$W_{T}^{ \pm} Z_{T}$ & 5755 & 164 & 12.0 \\
\hline$W_{L}^{+} W_{L}^{-}$ & $1416\left(1+\frac{0.0318}{\Lambda^{2}}+\frac{0.00203}{\Lambda^{4}}\right)$ & $34.0\left(1+\frac{0.597}{\Lambda^{2}}+\frac{0.121}{\Lambda^{4}}\right)$ & $2.75\left(1+\frac{1.83}{\Lambda^{2}}+\frac{1.05}{\Lambda^{4}}\right)$ \\
\hline$W_{L(T)}^{+} W_{T(L)}^{-}$ & $4866\left(1+\frac{0.00758}{\Lambda^{2}}+\frac{0.000489}{\Lambda^{4}}\right)$ & $34.6\left(1+\frac{0.130}{\Lambda^{2}}+\frac{0.0207}{\Lambda^{4}}\right)$ & $0.848\left(1+\frac{0.429}{\Lambda^{2}}+\frac{0.213}{\Lambda^{4}}\right)$ \\
\hline$W_{T}^{+} W_{T}^{-}$ & 17987 & 523 & 39.1 \\
\hline$W_{L}^{ \pm} h$ & $387\left(1+\frac{0.149}{\Lambda^{2}}+\frac{0.00776}{\Lambda^{4}}\right)$ & $46.5\left(1+\frac{0.712}{\Lambda^{2}}+\frac{0.148}{\Lambda^{4}}\right)$ & $4.30\left(1+\frac{2.13}{\Lambda^{2}}+\frac{1.24}{\Lambda^{4}}\right)$ \\
\hline$W_{T}^{ \pm} h$ & $270\left(1+\frac{0.0302}{\Lambda^{2}}+\frac{0.000146}{\Lambda^{4}}\right)$ & $4.93\left(1+\frac{0.0287}{\Lambda^{2}}+\frac{0.000217}{\Lambda^{4}}\right)$ & $0.140\left(1+\frac{0.0271}{\Lambda^{2}}+\frac{0.000275}{\Lambda^{4}}\right)$ \\
\hline$Z_{L} h$ & $198\left(1+\frac{0.134}{\Lambda^{2}}+\frac{0.00731}{\Lambda^{4}}\right)$ & $24.5\left(1+\frac{0.628}{\Lambda^{2}}+\frac{0.136}{\Lambda^{4}}\right)$ & $2.24\left(1+\frac{1.90}{\Lambda^{2}}+\frac{1.14}{\Lambda^{4}}\right)$ \\
\hline$Z_{T} h$ & $154\left(1+\frac{0.0361}{\Lambda^{2}}+\frac{0.000353}{\Lambda^{4}}\right)$ & $3.30\left(1+\frac{0.0688}{\Lambda^{2}}+\frac{0.00501}{\Lambda^{4}}\right)$ & $0.0941\left(1+\frac{0.165}{\Lambda^{2}}+\frac{0.0413}{\Lambda^{4}}\right)$ \\
\hline$\sigma[\mathrm{fb}], p_{T}[\mathrm{GeV}]$ & {$[600,800]$} & {$[800,1000]$} & {$[1000,1500]$} \\
\hline$W_{L}^{ \pm} Z_{L}$ & $0.799\left(1+\frac{4.30}{\Lambda^{2}}+\frac{4.87}{\Lambda^{4}}\right)$ & $0.188\left(1+\frac{6.92}{\Lambda^{2}}+\frac{13.4}{\Lambda^{4}}\right)$ & $0.0749\left(1+\frac{11.9}{\Lambda^{2}}+\frac{39.1}{\Lambda^{4}}\right)$ \\
\hline$W_{L(T)}^{ \pm} Z_{T(L)}$ & $0.0471\left(1+\frac{2.91}{\Lambda^{2}}+\frac{2.60}{\Lambda^{4}}\right)$ & $0.00634\left(1+\frac{4.89}{\Lambda^{2}}+\frac{7.34}{\Lambda^{4}}\right)$ & $0.00149\left(1+\frac{8.01}{\Lambda^{2}}+\frac{20.6}{\Lambda^{4}}\right)$ \\
\hline$W_{T}^{ \pm} Z_{T}$ & 1.74 & 0.357 & 0.121 \\
\hline$W_{L}^{+} W_{L}^{-}$ & $0.442\left(1+\frac{3.74}{\Lambda^{2}}+\frac{4.13}{\Lambda^{4}}\right)$ & $0.102\left(1+\frac{6.13}{\Lambda^{2}}+\frac{11.4}{\Lambda^{4}}\right)$ & $0.0405\left(1+\frac{10.4}{\Lambda^{2}}+\frac{32.6}{\Lambda^{4}}\right)$ \\
\hline$W_{L(T)}^{+} W_{T(L)}^{-}$ & $0.0652\left(1+\frac{0.888}{\Lambda^{2}}+\frac{0.889}{\Lambda^{4}}\right)$ & $0.00873\left(1+\frac{1.49}{\Lambda^{2}}+\frac{2.47}{\Lambda^{4}}\right)$ & $0.00204\left(1+\frac{2.43}{\Lambda^{2}}+\frac{6.79}{\Lambda^{4}}\right)$ \\
\hline$W_{T}^{+} W_{T}^{-}$ & 5.92 & 1.28 & 0.475 \\
\hline$W_{L}^{ \pm} h$ & $0.726\left(1+\frac{4.22}{\Lambda^{2}}+\frac{4.83}{\Lambda^{4}}\right)$ & $0.169\left(1+\frac{7.15}{\Lambda^{2}}+\frac{13.3}{\Lambda^{4}}\right)$ & $0.0671\left(1+\frac{12.2}{\Lambda^{2}}+\frac{38.7}{\Lambda^{4}}\right)$ \\
\hline$W_{T}^{ \pm} h$ & $0.0112\left(1+\frac{0.0283}{\Lambda^{2}}+\frac{0.000218}{\Lambda^{4}}\right)$ & 0.00153 & 0.000364 \\
\hline$Z_{L} h$ & $0.367\left(1+\frac{3.82}{\Lambda^{2}}+\frac{4.50}{\Lambda^{4}}\right)$ & $0.0835\left(1+\frac{6.27}{\Lambda^{2}}+\frac{12.4}{\Lambda^{4}}\right)$ & $0.0327\left(1+\frac{11.0}{\Lambda^{2}}+\frac{35.9}{\Lambda^{4}}\right)$ \\
\hline$Z_{T} h$ & $0.00737\left(1+\frac{0.318}{\Lambda^{2}}+\frac{0.165}{\Lambda^{4}}\right)$ & $0.000991\left(1+\frac{0.523}{\Lambda^{2}}+\frac{0.455}{\Lambda^{4}}\right)$ & $0.000231\left(1+\frac{0.838}{\Lambda^{2}}+\frac{1.24}{\Lambda^{4}}\right)$ \\
\hline
\end{tabular}




\section{APPENDIX B: POLARIZATION MEASUREMENT OF THE $W$ BOSON}

To measure the $W$ polarization, we need to study the angular distribution of its decay products. We choose the polarization axis to be the direction of the $W$ in the laboratory frame. The amplitude for the $W^{+}\left(p_{W}\right) \rightarrow l^{+}\left(p_{\ell}\right) \nu\left(p_{\nu}\right)$ is

$$
\mathcal{M}=\frac{g}{\sqrt{2}} \bar{u}_{L}\left(p_{\nu}\right) \gamma^{\mu} \nu_{L}\left(p_{\ell}\right) \epsilon_{\mu}^{*}\left(p_{W}\right)
$$

Let us start from the rest frame of the $W^{+}$. We parametrize the momenta of leptons as follows:

$$
\begin{aligned}
& p_{\ell}^{*}=(k, \vec{k})=\left(k, k \sin \theta^{*} \cos \varphi^{*}, k \sin \theta^{*} \sin \varphi^{*}, k \cos \theta^{*}\right), \\
& p_{\nu}^{*}=(k,-\vec{k})=\left(k,-k \sin \theta^{*} \cos \varphi^{*},-k \sin \theta^{*} \sin \varphi^{*},-k \cos \theta^{*}\right),
\end{aligned}
$$

where $k=|\vec{k}|=m_{W} / 2$. The general expressions for the helicity spinors are given by

$$
\begin{array}{ll}
\xi_{L}\left(p_{\ell}^{*}\right)=\left(\begin{array}{cc}
-e^{-i \varphi^{*} / 2} \sin \frac{\theta^{*}}{2} \\
e^{i \varphi^{*} / 2} \cos \frac{\theta^{*}}{2}
\end{array}\right), & \xi_{R}\left(p_{\ell}^{*}\right)=\left(\begin{array}{c}
e^{-i \varphi^{*} / 2} \cos \frac{\theta^{*}}{2} \\
e^{i \varphi^{*} / 2} \sin \frac{\theta^{*}}{2}
\end{array}\right) \\
\xi_{L}\left(p_{\nu}^{*}\right)=\left(\begin{array}{c}
e^{-i \varphi^{*} / 2} \cos \frac{\theta^{*}}{2} \\
e^{i \varphi^{*} / 2} \sin \frac{\theta^{*}}{2}
\end{array}\right), & \xi_{R}\left(p_{\nu}^{*}\right)=\left(\begin{array}{c}
-e^{-i \varphi^{*} / 2} \sin \frac{\theta^{*}}{2} \\
e^{i \varphi^{*} / 2} \cos \frac{\theta^{*}}{2}
\end{array}\right)
\end{array}
$$

and the left-handed current is as follows:

$$
\bar{u}_{L}\left(p_{\nu}^{*}\right) \gamma^{\mu} \nu_{L}\left(p_{\ell}^{*}\right)=2 k\left(0,-e^{i \varphi^{*}} \cos ^{2} \frac{\theta^{*}}{2}+e^{-i \varphi^{*}} \sin ^{2} \frac{\theta^{*}}{2}, i e^{i \varphi^{*}} \cos ^{2} \frac{\theta^{*}}{2}+i e^{-i \varphi^{*}} \sin ^{2} \frac{\theta^{*}}{2}, \sin \theta^{*}\right)^{\mu} .
$$

By using the formulas of the polarization vectors,

$$
\epsilon^{+\mu}=\frac{1}{\sqrt{2}}\left(\begin{array}{c}
0 \\
1 \\
i \\
0
\end{array}\right), \quad \epsilon^{-\mu}=\frac{1}{\sqrt{2}}\left(\begin{array}{c}
0 \\
1 \\
-i \\
0
\end{array}\right), \quad \epsilon^{0 \mu}=\left(\begin{array}{l}
0 \\
0 \\
0 \\
1
\end{array}\right),
$$

we can easily obtain the helicity amplitudes:

$$
\mathcal{M}^{+}=-g k e^{-i \varphi^{*}}\left(1-\cos \theta^{*}\right), \quad \mathcal{M}^{-}=g k e^{i \varphi^{8}}\left(1+\cos \theta^{*}\right), \quad \mathcal{M}^{0}=-\sqrt{2} g k \sin \theta^{*},
$$

which leads to the distribution in Eq. (15). Turn to the laboratory frame and suppose that we can reconstruct the $z$-momentum of the neutrino by imposing the condition that the lepton-neutrino system should correctly reproduce the mass of the $W$ boson. The momentum of the charged lepton and neutrino in the laboratory frame can be obtained from the momentum in the $W^{+}$rest frame by a Lorentz boost:

$$
\begin{array}{ll}
E_{\ell}=\gamma(k+\vec{v} \cdot \vec{k}), & \vec{p}_{\ell}=\vec{k}+\vec{v}\left(\gamma k+\frac{\gamma-1}{v^{2}} \vec{v} \cdot \vec{k}\right) \\
E_{\nu}=\gamma(k-\vec{v} \cdot \vec{k}), & \vec{p}_{\nu}=-\vec{k}+\vec{v}\left(\gamma k-\frac{\gamma-1}{v^{2}} \vec{v} \cdot \vec{k}\right),
\end{array}
$$

where $\vec{v}=\frac{\vec{p}_{W}}{E_{W}}, v=|\vec{v}|$ is the velocity of the $W^{+}$in the laboratory frame. Then the formulas of $\cos \theta^{*}$ can be obtained by the energy difference of the lepton and neutrino in the laboratory frame as follows:

$$
\cos \theta^{*}=\frac{\vec{v} \cdot \vec{k}}{k v}=\frac{E_{\ell}-E_{\nu}}{\left|\vec{p}_{W}\right|}=\frac{\left|\vec{p}_{\ell}\right|-\left|\vec{p}_{\nu}\right|}{\left|\vec{p}_{\ell}+\vec{p}_{\nu}\right|},
$$

where we have used

$$
k=\frac{m_{W}}{2}, \quad\left|\vec{p}_{W}\right|=m_{W} \gamma v
$$


[1] B. Grzadkowski, M. Iskrzynski, M. Misiak, and J. Rosiek, Dimension-six terms in the Standard Model Lagrangian, J. High Energy Phys. 10 (2010) 085.

[2] J. Elias-Miro, J. R. Espinosa, E. Masso, and A. Pomarol, Higgs windows to new physics through $d=6$ operators: Constraints and one-loop anomalous dimensions, J. High Energy Phys. 11 (2013) 066.

[3] R. Contino, M. Ghezzi, C. Grojean, M. Muhlleitner, and M. Spira, Effective Lagrangian for a light Higgs-like scalar, J. High Energy Phys. 07 (2013) 035.

[4] LEP Electroweak Working Group, Precision electroweak measurements and constraints on the Standard Model, arXiv:1012.2367.

[5] J. Ellis, V. Sanz, and T. You, Complete Higgs sector constraints on dimension-6 operators, J. High Energy Phys. 07 (2014) 036.

[6] A. Falkowski and F. Riva, Model-independent precision constraints on dimension-6 operators, J. High Energy Phys. 02 (2015) 039.

[7] A. Falkowski, M. Gonzalez-Alonso, A. Greljo, and D. Marzocca, Global Constraints on Anomalous Triple Gauge Couplings in Effective Field Theory Approach, Phys. Rev. Lett. 116, 011801 (2016).

[8] A. Butter, O. J. P. Éboli, J. Gonzalez-Fraile, M. C. Gonzalez-Garcia, T. Plehn, and M. Rauch, The GaugeHiggs legacy of the LHC run I, J. High Energy Phys. 07 (2016) 152.

[9] Z. Zhang, Time to Go Beyond Triple-Gauge-BosonCoupling Interpretation of $W$ Pair Production, Phys. Rev. Lett. 118, 011803 (2017).

[10] A. Falkowski, M. Gonzalez-Alonso, A. Greljo, D. Marzocca, and M. Son, Anomalous triple gauge couplings in the effective field theory approach at the LHC, J. High Energy Phys. 02 (2017) 115.

[11] D. R. Green, P. Meade, and M.-A. Pleier, Multiboson interactions at the LHC, Rev. Mod. Phys. 89, 035008 (2017).

[12] A. Biekötter, A. Knochel, M. Krämer, D. Liu, and F. Riva, Vices and virtues of Higgs effective field theories at large energy, Phys. Rev. D 91, 055029 (2015).

[13] J. Baglio, S. Dawson, and I. M. Lewis, An NLO QCD effective field theory analysis of $W^{+} W^{-}$production at the LHC including fermionic operators, Phys. Rev. D 96, 073003 (2017).

[14] J. Ellis, C. W. Murphy, V. Sanz, and T. You, Updated global SMEFT fit to Higgs, Diboson and electroweak data, J. High Energy Phys. 06 (2018) 146.

[15] R. Franceschini, G. Panico, A. Pomarol, F. Riva, and A. Wulzer, Electroweak precision tests in high-energy diboson processes, J. High Energy Phys. 02 (2018) 111.

[16] M. Chiesa, A. Denner, and J.-N. Lang, Anomalous triplegauge-boson interactions in vector-boson pair production with RECOLA2, Eur. Phys. J. C 78, 467 (2018).

[17] J. M. Butterworth, I. Ochoa, and T. Scanlon, Boosted Higgs $\rightarrow b \bar{b}$ in vector-boson associated production at 14 TeV, Eur. Phys. J. C 75, 366 (2015).

[18] F. Tian, Combined analysis of jet substructure for Higgs decay to $b \bar{b}$ in vector boson associated production at the $13 \mathrm{TeV}$ LHC, arXiv:1701.08413.

[19] V. Sanz and J. Setford, Composite Higgs models after run 2, Adv. High Energy Phys. 2018, 7168480 (2018).
[20] J. de Blas, O. Eberhardt, and C. Krause, Current and future constraints on Higgs couplings in the nonlinear effective theory, J. High Energy Phys. 07 (2018) 048.

[21] CMS Collaboration, CMS at the high-energy Frontier. Contribution to the update of the European strategy for particle physics, Technical Report, CERN Reports No. CMS-NOTE-2012-006, No. CERN-CMS-NOTE2012-006.

[22] ATLAS Collaboration, Projections for measurements of Higgs boson cross sections, branching ratios and coupling parameters with the ATLAS detector at a HL-LHC, Technical Report No. ATL-PHYS-PUB-2013-014, CERN, Geneva, 2013, http://cds.cern.ch/record/1611186.

[23] S. Dawson et al., Working group report: Higgs boson, arXiv:1310.8361, https://inspirehep.net/record/1262795/files/ arXiv:1310.8361.pdf.

[24] M. S. Chanowitz and M. K. Gaillard, The TeV physics of strongly interacting $W$ 's and Z's, Nucl. Phys. B261, 379 (1985).

[25] A. Wulzer, An equivalent gauge and the equivalence theorem, Nucl. Phys. B885, 97 (2014).

[26] R. Contino, C. Grojean, M. Moretti, F. Piccinini, and R. Rattazzi, Strong double Higgs production at the LHC, J. High Energy Phys. 05 (2010) 089.

[27] M. Szleper, The Higgs boson and the physics of $W W$ scattering before and after Higgs discovery, arXiv:1412.8367.

[28] S. Fichet, A. Tonero, and P. Rebello Teles, Sharpening the shape analysis for higher-dimensional operator searches, Phys. Rev. D 96, 036003 (2017).

[29] J. M. Butterworth, A. R. Davison, M. Rubin, and G. P. Salam, Jet Substructure as a New Higgs Search Channel at the LHC, Phys. Rev. Lett. 100, 242001 (2008).

[30] A. J. Larkoski, I. Moult, and B. Nachman, Jet substructure at the large Hadron collider: A review of recent advances in theory and machine learning, arXiv:1709.04464.

[31] L. Asquith et al., Jet substructure at the large Hadron collider: Experimental review, arXiv:1803.06991.

[32] K. Hagiwara, R. D. Peccei, D. Zeppenfeld, and K. Hikasa, Probing the weak boson sector in $e^{+} e^{-} \rightarrow W^{+} W^{-}$, Nucl. Phys. B282, 253 (1987).

[33] A. Azatov, R. Contino, C. S. Machado, and F. Riva, Helicity selection rules and noninterference for BSM amplitudes, Phys. Rev. D 95, 065014 (2017).

[34] U. Baur, T. Han, and J. Ohnemus, Amplitude Zeros in $W^{ \pm} Z$ Production, Phys. Rev. Lett. 72, 3941 (1994).

[35] C. Frye, M. Freytsis, J. Scholtz, and M. J. Strassler, Precision diboson observables for the LHC, J. High Energy Phys. 03 (2016) 171.

[36] T. Han, D. Krohn, L.-T. Wang, and W. Zhu, New physics signals in longitudinal gauge boson scattering at the LHC, J. High Energy Phys. 03 (2010) 082.

[37] D. Liu, A. Pomarol, R. Rattazzi, and F. Riva, Patterns of strong coupling for LHC searches, J. High Energy Phys. 11 (2016) 141.

[38] M.E. Peskin and T. Takeuchi, Estimation of oblique electroweak corrections, Phys. Rev. D 46, 381 (1992).

[39] A. Alloul, N. D. Christensen, C. Degrande, C. Duhr, and B. Fuks, FeynRules 2.0: A complete toolbox for tree-level phenomenology, Comput. Phys. Commun. 185, 2250 (2014). 
[40] J. Alwall, R. Frederix, S. Frixione, V. Hirschi, F. Maltoni, O. Mattelaer, H. S. Shao, T. Stelzer, P. Torrielli, and M. Zaro, The automated computation of tree-level and next-toleading order differential cross sections, and their matching to parton shower simulations, J. High Energy Phys. 07 (2014) 079.

[41] R. D. Ball et al., Parton distributions with LHC data, Nucl. Phys. B867, 244 (2013).

[42] CMS Collaboration, CMS detector performance during LHC run 1 and projections for run 2, Nucl. Part. Phys. Proc. 273-275, 1048 (2016).

[43] ATLAS Collaboration, Expected performance of the ATLAS experiment-detector, Trigger and physics, arXiv:0901 .0512 .

[44] ATLAS and T. A. Collaborations, Electron efficiency measurements with the ATLAS detector using the 2015 LHC proton-proton collision data.

[45] G. Aad et al. (ATLAS Collaboration), Muon reconstruction performance of the ATLAS detector in proton-proton collision data at $\sqrt{s}=13 \mathrm{TeV}$, Eur. Phys. J. C 76, 292 (2016).

[46] CMS Collaboration, Identification techniques for highly boosted $W$ bosons that decay into hadrons, J. High Energy Phys. 12 (2014) 017.

[47] CMS Collaboration, Search for anomalous couplings in boosted $W W / W Z \rightarrow \ell \nu q \bar{q}$ production in proton-proton collisions at $\sqrt{s}=8 \mathrm{TeV}$, Phys. Lett. B 772, 21 (2017).

[48] ATLAS Collaboration, Measurement of $W W / W Z \rightarrow \ell \nu q q^{\prime}$ production with the hadronically decaying boson reconstructed as one or two jets in $p p$ collisions at $\sqrt{s}=8 \mathrm{TeV}$ with ATLAS, and constraints on anomalous gauge couplings, Eur. Phys. J. C 77, 563 (2017).

[49] ATLAS Collaboration, Performance of top quark and $W$ boson tagging in run 2 with ATLAS, Report No. ATLASCONF-2017-064, 2017.

[50] ATLAS Collaboration, Identification of boosted, hadronically-decaying $W$ and $Z$ bosons in $\sqrt{s}=13 \mathrm{TeV}$ Monte Carlo simulations for ATLAS, Technical Report No. ATL-PHYS-PUB-2015-033, CERN, Geneva, http:// cds.cern.ch/record/2041461.
[51] R. Contino, A. Falkowski, F. Goertz, C. Grojean, and F. Riva, On the validity of the effective field theory approach to SM precision tests, J. High Energy Phys. 07 (2016) 144.

[52] A. Azatov, J. Elias-Miro, Y. Reyimuaji, and E. Venturini, Novel measurements of anomalous triple gauge couplings for the LHC, J. High Energy Phys. 10 (2017) 027.

[53] G. Panico, F. Riva, and A. Wulzer, Diboson interference resurrection, Phys. Lett. B 776, 473 (2018).

[54] R. Barbieri, A. Pomarol, R. Rattazzi, and A. Strumia, Electroweak symmetry breaking after LEP-1 and LEP-2, Nucl. Phys. B703, 127 (2004).

[55] A. Pomarol and F. Riva, Towards the ultimate SM fit to close in on Higgs physics, J. High Energy Phys. 01 (2014) 151.

[56] J. Ellis, V. Sanz, and T. You, The effective Standard Model after LHC run I, J. High Energy Phys. 03 (2015) 157.

[57] M. Farina, G. Panico, D. Pappadopulo, J. T. Ruderman, R. Torre, and A. Wulzer, Energy helps accuracy: Electroweak precision tests at hadron colliders, Phys. Lett. B 772, 210 (2017).

[58] G. F. Giudice, C. Grojean, A. Pomarol, and R. Rattazzi, The strongly-interacting light Higgs, J. High Energy Phys. 06 (2007) 045.

[59] K. Agashe, R. Contino, and A. Pomarol, The minimal composite Higgs model, Nucl. Phys. B719, 165 (2005).

[60] D. B. Kaplan, Flavor at SSC energies: A new mechanism for dynamically generated fermion masses, Nucl. Phys. B365, 259 (1991).

[61] G. D’Ambrosio, G. F. Giudice, G. Isidori, and A. Strumia, Minimal flavor violation: An effective field theory approach, Nucl. Phys. B645, 155 (2002).

[62] ATLAS Collaboration, Search for new phenomena in dijet events with the ATLAS detector at $\sqrt{s}=13 \mathrm{TeV}$ with 2015 and 2016 data, Report No. ATLAS-CONF-2016-069.

[63] B. Bellazzini, F. Riva, J. Serra, and F. Sgarlata, The other effective fermion compositeness, J. High Energy Phys. 11 (2017) 020.

[64] O. Domenech, A. Pomarol, and J. Serra, Probing the SM with Dijets at the LHC, Phys. Rev. D 85, 074030 (2012).

[65] S. Alioli, M. Farina, D. Pappadopulo, and J. T. Ruderman, Precision probes of QCD at high energies, J. High Energy Phys. 07 (2017) 097. 